\title{
MicroRNA and mRNA analysis of angiotensin II-induced renal artery endothelial cell dysfunction
}

\author{
YAO LIU ${ }^{1}$, YUEHUA JIANG $^{2}$, WEI LI $^{3}$, CONG HAN $^{1}$ and ZHENQIANG QI ${ }^{1}$ \\ ${ }^{1}$ Department of Clinical Chinese Medicine integrated with Western Medicine, First Clinical Medical \\ College, Shandong University of Traditional Chinese Medicine; \\ ${ }^{2}$ Central Laboratory; ${ }^{3}$ Nephropathy Department, Affiliated Hospital of Shandong University of \\ Traditional Chinese Medicine, Jinan, Shandong 250014, P.R. China
}

Received July 8, 2019; Accepted January 17, 2020

DOI: $10.3892 /$ etm.2020.8613

\begin{abstract}
Continuous activation of angiotensin II (Ang II) induces renal vascular endothelial dysfunction, inflammation and oxidative stress, all of which may contribute to renal damage. MicroRNAs (miRs/miRNAs) play a crucial regulatory role in the pathogenesis of hypertensive nephropathy (HN). The present study aimed to assess the differential expression profiles of potential candidate genes involved in Ang II-induced rat renal artery endothelial cell (RRAEC) dysfunction and explore their possible functions. In the present study, the changes in energy metabolism and autophagy function in RRAECs were evaluated using the Seahorse XF Glycolysis Stress Test and dansylcadaverine/transmission electron microscopy following exposure to Ang II. Subsequently, mRNA-miRNA sequencing experiments were performed to determine the differential expression profiles of mRNAs and miRNAs. Integrated bioinformatics analysis was applied to further explore the molecular mechanisms of Ang II on endothelial injury induced by Ang II. The present
\end{abstract}

Correspondence to: Professor Wei Li, Nephropathy Department, Affiliated Hospital of Shandong University of Traditional Chinese Medicine, Jinan, Shandong 250014, P.R. China

E-mail: 1weidw@163.com

Abbreviations: Ang II, Angiotensin II; HN, hypertensive nephropathy; RRAEC, rat renal artery endothelial cell; DE, differentially expressed; RAS, renin-angiotensin system; ET-1, endothelin-1; NO, nitric oxide; ECAR, extracellular acidification rate; MDC, dansylcadaverine; TEM, transmission electron microscopy; FC, fold change; RT-qPCR, reverse transcription-quantitative PCR; GO, Gene ontology; CC, cellular component; MF, molecular function; BP, biological process; MMP-28, matrix metalloproteinase-28; ANO1, anoctamin-1; Mylk2, myosin light chain kinase 2; MLCK, myosin light chain kinase; Hsd11b2, hydroxysteroid dehydrogenase11b2; ECs, endothelial cells; Pfkfb3, 6-phosphofructo-2-kinase/fructose-2,6-biphosphatase 3

Key words: microRNA, mRNA, angiotensin II, renal artery endothelial dysfunction, hypertensive nephropathy data supported the notion that Ang II upregulated glycolysis levels and promoted autophagy activation in RRAECs. The sequencing data demonstrated that 443 mRNAs and 58 miRNAs were differentially expressed (DE) in response to Ang II exposure, where 66 mRNAs and 55 miRNAs were upregulated, while 377 mRNAs and 3 miRNAs were downregulated (fold change $>1.5$ or $<0.67 ; \mathrm{P}<0.05$ ). Functional analysis indicated that DE mRNA and DE miRNA target genes were mainly associated with cell metabolism (metabolic pathways), differentiation (Th1 and Th2 cell differentiation), autophagy (autophagy-animal and autophagy-other) and repair (RNA-repair). To the best of the authors' knowledge, this is the first report on mRNA-miRNA integrated profiles of Ang II-induced RRAECs. The present results provided evidence suggesting that the miRNA-mediated effect on the 'mTOR signaling pathway' might play a role in Ang II-induced RRAEC injury by driving glycolysis and autophagy activation. Targeting miRNAs and their associated pathways may provide valuable insight into the clinical management of HN and may improve patient outcome.

\section{Introduction}

Recent studies have found that the local renin-angiotensin system (RAS) might contribute to glomerulosclerosis and renal interstitial fibrosis (1). The organ-specific roles exerted by angiotensin II (Ang II), which is the most potent biologically active product of the RAS, have also been studied (2). The RAS is overactivated in vivo once hypertension occurs. Overproduced Ang II directly constricts vascular endothelial cells (ECs), causes changes in diastolic and contractile substances, increases the synthesis and release of endothelium-derived vasoconstrictors such as endothelin-1 (ET-1) and thromboxane A2, reduces the production of endothelium-derived vasodilators such as nitric oxide (NO) and ultimately results in vascular endothelial damage and retention of sodium and water $(3,4)$. Further studies demonstrated that inappropriate activation of intrarenal Ang II plays a central role in the pathogenesis of hypertension and renal injury (5). The role of renal artery ECs in self-regulation is associated with cell autophagy and energy homeostasis. However, activation of the endothelium by elevated blood pressure is followed 
by endothelial dysfunction, which eventually leads to endothelial disintegration (6). In this context, dysfunctional ECs may continue generating ATP by glycolysis for a long period of time and keep their mitochondrial membrane potential in a depolarized state that can be reverted. Furthermore, under these circumstances, the autophagy pathway may be activated to maintain glycolytic-dependent ATP production (7). The effect of hypertension on renal vascular endothelium is directly related to hypertensive nephropathy (HN), but few studies have harnessed the power of transcriptome sequencing or microarray analysis to identify the potential vulnerabilities of hypertensive renal artery injury. Therefore, the molecular mechanism of Ang II on renal artery ECs has important research value and significance.

MicroRNAs (miRs/miRNAs) are post-transcriptional regulators of gene expression. These small (20-25 nucleotides long) noncoding RNAs bind to a target recognition site (seed sequence) in the 3'-untranslated regions of mRNA transcripts, leading to mRNA degradation and/or inhibition or activation of protein translation, depending on the complementarity of the miRNA with the target Mrna (8). A growing number of miRNAs, including miRNA-let-7b, miRNA-431 and miRNA-29 (9-11), are implicated in the promotion or suppression in the initiation and progression of hypertension. Ang II-mediated STAT3 activation in kidney epithelial cells results in hypertensive kidney disease (12); however, the detailed mechanisms and regulatory role as therapeutic targets of miRNAs underlying renal artery EC injury induced by Ang II remain poorly understood. Hence, the present study focused on the miRNAs involved in renal artery EC dysfunction.

The present study constructed an Ang II-induced rat renal artery EC (RRAEC) injury model. The mitochondrial membrane potential and glycolysis levels were determined to assess mitochondrial function and cellular energy supply of RRAECs exposed to Ang II. The stability of the intracellular environment was evaluated by measuring the level of autophagy. Subsequently, mRNA and miRNA sequencing and integrated analysis of differentially expressed (DE) miRNAs and their target mRNAs were performed to further explore the precise molecular mechanisms responsible for Ang II-induced renal artery endothelial dysfunction. The results of the present study could provide new insights into the potential therapeutic targets of HN.

\section{Materials and methods}

Cell culture and reagents. RRAECs (cat. no. RAEC; Sixin) were cultured in DMEM (cat. no. 10-013-CVR; Corning, Inc.) supplemented with 10\% FBS (cat. no. 10099-141; Gibco; Thermo Fisher Scientific, Inc.) and $1 \%$ penicillin/streptomycin (cat. no. SV30010; HyClone; GE Healthcare Life Sciences) at $37^{\circ} \mathrm{C}$ and $5 \% \mathrm{CO}_{2}$. Cells from passages 2-8 were used for subsequent experiments. RRAECs were seeded in six-well plates at a density of $2 \times 10^{4}$ cells/well for $24 \mathrm{~h}$ with exposure to

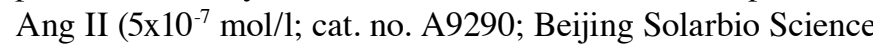
\& Technology Co., Ltd.) at $37^{\circ} \mathrm{C}$, while untreated RRAECs were used as a negative control. The cells were serum-starved overnight before further treatment. All samples were assayed in triplicate.
Detection of mitochondrial membrane potential. JC-1 is a mitochondrion-selective dye widely used to detect mitochondrial membrane potential $(\Delta \Psi \mathrm{m})$, which reversibly changes color from green to red as the membrane potential increases (values of $>80-100 \mathrm{mv}$ ). The degree of mitochondrial depolarization is measured by the relative proportion of red and green fluorescence (13). Following $24 \mathrm{~h}$ of Ang II exposure as described above, the cells were collected and washed with PBS (cat. no. 21-040-CVR; Corning, Inc.). Washed cells were resuspended in JC-1 staining solution (cat. no. M8650; Beijing Solarbio Science \& Technology Co., Ltd.) and incubated at $37^{\circ} \mathrm{C}$ for $20 \mathrm{~min}$. RRAECs were then washed twice with the diluted working solution (JC-1 staining solution; 1X). Mitochondrial membrane potential analysis was performed using a flow cytometer (BD Biosciences) and analyzed by CytExpert for DXFLEX 2.0 (Beckman Coulter, Inc.).

Seahorse XF analysis. The Seahorse XF Glycolysis Stress Test performed using the Seahorse XF(e)24 Cell Energy Metabolic Analyzer (Agilent Technologies, Inc.) directly measures the extracellular acidification rate (ECAR) of living cells in real time, reflecting glycolytic function in cells. Cell density was optimized by inoculating different amounts of RRAECs (1-8x10 cells/well) into a 24-well Seahorse XF Cell Culture Microplate (cat. no. 100777-004; Agilent Technologies, Inc.) at $37^{\circ} \mathrm{C}$ and $5 \% \mathrm{CO}_{2}$. Subsequent assays were performed using $3 \times 10^{4}$ cells/well. A sensor cartridge was hydrated in a Seahorse XF Calibrant (Agilent Technologies, Inc.) at $37^{\circ} \mathrm{C}$ in a non- $\mathrm{CO}_{2}$ incubator overnight. XF Glycolysis Stress Test kit medium (cat. no. 103020-100; Agilent Technologies, Inc.) was heated to $37^{\circ} \mathrm{C}$, and the $\mathrm{pH}$ was adjusted to $7.4 \pm 0.1$. The cell culture microplate was removed from the $37^{\circ} \mathrm{C} \mathrm{CO}_{2}$ incubator, and the RRAECs were washed with XF glycolysis assay medium and then placed in a $37^{\circ} \mathrm{C}$, non- $\mathrm{CO}_{2}$ incubator for $1 \mathrm{~h}$ prior to the assay. Injection ports $\mathrm{A}, \mathrm{B}$, and $\mathrm{C}$ were loaded with glucose $(10 \mathrm{mmol} / \mathrm{l})$, oligomycin $(1 \mu \mathrm{mol} / \mathrm{l})$ and 2-deoxy-glucose $(50 \mathrm{mmol} / \mathrm{l}$; included in the Glycolysis Stress assay kit), according to the manufacturer's protocol. The experimental template was set up or imported on the XF controller. The default Mix-Wait-Measure time was 3 min-2 min-3 min. Seahorse Wave software (version 2.2.0; Agilent Technologies, Inc.) was used for data calculation. The XF Glycolysis Stress Test was used to measure glycolysis as the ECAR that is reached by RRAECs after the addition of saturating amounts of glucose. The glycolytic reserve was defined as the difference between glycolytic capacity and glycolysis rate. Prior to glucose injection, ECAR was referred to as non-glycolytic acidification and was caused by processes in the cell other than glycolysis.

Autophagy rate assay. The effect of Ang II on autophagy rate was assessed with a dansylcadaverine (MDC) Autophagy Assay kit (cat. no. MAK138-1KT; Sigma-Aldrich; Merck $\mathrm{KGaA}$ ) by flow cytometry. MDC is an acidic vesicle-specific fluorescent marker that directly reflects the number of acidic vesicles in the cells (14). RRAECs were treated with Ang II for $24 \mathrm{~h}$ and then trypsinized and collected. Cells $\left(5 \times 10^{5}\right)$ were washed with PBS twice, incubated with MDC staining solution at $37^{\circ} \mathrm{C}$ for $1 \mathrm{~h}$ and then incubated with $4 \%$ paraformaldehyde at room temperature for $15 \mathrm{~min}$. The stained cells 
were detected with a FACScan flow cytometer at a wavelength of $488 \mathrm{~nm}$, and the total autophagy rate (without distinguishing between macroautophagy and mitochondrial autophagy) was statistically analyzed. Data were analyzed using cell Quest 3.1 software (Becton, Dickinson and Company).

Observation of autophagosomes by transmission electron microscopy (TEM). As the most reliable method and gold standard for detecting phagocytosis (15), TEM was used to clearly observe the independent bilayer membrane structure of autophagosomes and various autophagic morphological changes at x10,000 magnification. Autophagosomes in the control group and the Ang II group were observed by TEM (JEOL, Ltd.). RRAECs at a density of $1 \times 10^{5}$ cells/well were seeded in six-well plates with or without exposure to Ang II for $24 \mathrm{~h}$. Subsequently, the cells were gently scraped and centrifuged at a rate of $2,000 \mathrm{x}$ g for $10 \mathrm{~min}$ at $37^{\circ} \mathrm{C}$ in PBS, following which RRAECs were fixed with $2.5 \%$ glutaraldehyde containing $0.1 \mathrm{~mol} / 1$ sodium cacodylate. Samples were fixed using $1 \%$ osmium tetroxide, followed by dehydration with an increasing concentration gradient of acetone solution. Samples were then embedded in epoxy resin provided by a SPI-PonTM 812 Epoxy Resin Embedding kit (cat. no. 02635-AB; SPI Supplies). The resin was subsequently polymerized at $37^{\circ} \mathrm{C}$ for $6 \mathrm{~h}, 45^{\circ} \mathrm{C}$ for $12 \mathrm{~h}$ and $67^{\circ} \mathrm{C}$ for $24 \mathrm{~h}$. All cell samples were then cut into $50-\mathrm{nm}$ sections and stained with $3 \%$ uranyl acetate for $10 \mathrm{~min}$ and lead citrate for $5 \mathrm{~min}$ at room temperature. Images were acquired using a TEM. Sample processing and image capture were performed by Weiya Biotechnology Co., Ltd.

RNA extraction and quality control. Total RNA was isolated using the miRNeasy Mini kit (Qiagen $\mathrm{GmbH}$ ), following which RNA concentration and quality were determined using a Qubit ${ }^{\circledR}$ 2.0 Fluorometer (Thermo Fisher Scientific, Inc.) and a NanoDrop ${ }^{\mathrm{TM}}$ One spectrophotometer (Thermo Fisher Scientific, Inc.). The integrity of total RNA was assessed using the Agilent 2100 Bioanalyzer (Agilent Technologies Inc.), and samples with RNA integrity number values $>7.0$ were used for sequencing.

Library construction for RNA-sequencing (RNA-seq) and sequencing procedures. Strand-specific libraries were prepared using the TruSeq ${ }^{\circledR}$ Stranded Total RNA Sample Preparation kit (Illumina, Inc.) following the manufacturer's instructions. Briefly, ribosomal RNA (rRNA) was removed from total RNA using Ribo-Zero rRNA removal beads. Following purification, the mRNA was fragmented into small pieces using divalent cations at $94^{\circ} \mathrm{C}$ for $8 \mathrm{~min}$. The cleaved RNA fragments were reverse transcribed into first strand cDNA using reverse transcriptase and random primers. This was followed by second strand cDNA synthesis using DNA Polymerase I and RNase $\mathrm{H}$. The cDNA fragments then went through an end repair process, the addition of a single 'A' base and adapter ligation. The products were then purified and amplified by PCR to create the final cDNA library. Purified libraries were quantified by a Qubit ${ }^{\circledR} 2.0$ Fluorometer (Thermo Fisher Scientific, Inc.) and validated with an Agilent 2100 Bioanalyzer (Agilent Technologies, Inc.) to confirm the insert size and to calculate the mole concentration. Clusters were generated by cBot, (Illumina, Inc.) with the library diluted to $10 \mathrm{pm}$ and sequenced on a HiSeq X Ten sequencer (Illumina, Inc.). Library construction and sequencing were performed at Shanghai Sinomics Corporation.

Library construction for miRNA-sequencing (miRNA-seq) and sequencing procedures. Paired-end libraries were synthesized using the QIAseq miRNA Library kit (Qiagen $\mathrm{GmbH}$ ) following the TruSeq ${ }^{\mathrm{TM}}$ RNA Sample Preparation Guide (QIAseq miRNA Library). The products were then purified and enriched with PCR to create the final cDNA library. Purified libraries were quantified by a Qubit ${ }^{\circledR} 2.0$ Fluorometer (Thermo Fisher Scientific, Inc.) and validated with an Agilent 2100 Bioanalyzer (Agilent Technologies, Inc.) to confirm the insert size and calculate the molar concentration. Clusters were generated by cBot (Illumina, Inc.) with the library diluted to $10 \mathrm{pm}$ and then sequenced on the Illumina HiSeq $\mathrm{X}^{\mathrm{TM}}$ Ten sequencer (Illumina, Inc.). Library construction and sequencing were performed at Sinotech Genomics Co., Ltd.).

Data analysis for gene expression. RNA-seq raw reads were pre-processed by filtering out rRNA reads, sequencing adapters, short-fragment reads and other low-quality reads. Tophat version 2.0.9 (https://ccb.jhu.edu/software/tophat/index.shtml) was used to map the clean reads to the Rnor_6.0.91 reference genome [Rnor_6.0 (GCA_000001895.4)] using alignment with two mismatches. Following genome mapping, Cufflinks version 2.1.1 (http://cole-trapnell-lab.github.io/cufflinks/) was run with a reference annotation to generate fragments per kilobase of exon model per million reads mapped (FPKM) values for known gene models. Differentially expressed genes were identified using edgeR package. The P-value significance threshold in multiple tests was set by the false discovery rate. The fold changes (FCs) were also estimated according to the FPKM of each sample. The differentially expressed genes were selected using the following filtering criteria: $\mathrm{P}<0.05$ and FC $>1.5$ or $<0.67$. Different samples and genes were clustered and classified and a heatmap was generated to display the expression of genes in different samples using $\mathrm{MeV} 4$ 4_6_0 software (multiple experiment viewer). Gene Ontology (GO) (http://geneontology.org/) adapted Fisher test, cluster profiler packages in R-3.4.3 and Bioconductor $3.6(16,17)$ and Kyoto Encyclopaedia of Genes and Genomes (KEGG) (https://www. kegg.jp/) pathway analysis were used to analyze the functions of the genes in the pathways. Reads of each sample containing miRNA sequences were compared with existing sequences in a miRNA database (miRBase; http://www.mirbase.org/) and the predicted results of new miRNAs generated by MiRDeep2 version 2.0.0.5 software to calculate the miRNA expression level (count number). miRNA expression was screened by counts per million. DE sequencing software (DESeq 1.30.0; https://bioconductor.org/packages/DESeq/) was used to analyze the expression between samples and identify DE miRNAs with $\mathrm{P}<0.05$ and $\mathrm{FC}>1.5$ or $<0.67$.

Reverse transcription-quantitative PCR (RT-qPCR) analysis of miRNA and mRNA expression. To verify the sequencing results, $15 \mathrm{DE}$ genes closely related to endothelial dysfunction were selected for RT-qPCR analysis: Nine miRNAs (miR-200a-3p, miR-200b-3p, miR-200c-3p, miR-192-5p, 
Table I. Primer sequences of miRNAs and mRNAs used in the study.

A, miRNA

\begin{tabular}{llc}
\hline Target & \multicolumn{1}{c}{ Forward sequence $\left(5^{\prime}-3^{\prime}\right)$} & Reverse sequence $\left(5^{\prime}-3^{\prime}\right)$ \\
\hline $\begin{array}{l}\text { rno-miR-29b-3p } \\
\text { rno-miR-200b-3p }\end{array}$ & TAGCACCATTTGAAATCAGTGTT & Universal \\
rno-miR-192-5p & TAATACTGCCTGGTAATGATGAC & CTGACCTATGAATTGACAGCC \\
rno-miR-223-3p & TGTCAGTTTGTCAAATACCCC \\
rno-miR-194-5p & TGTAACAGCAACTCCATGTGGA \\
rno-miR-200a-3p & TAACACTGTCTGGTAACGATGT \\
rno-miR-494-3p & TGAAACATACACGGGAAACCTCT \\
rno-miR-429 & TAATACTGTCTGGTAATGCCGT \\
rno-miR-200c-3p & TAATACTGCCGGGTAATGATG \\
U6 & GGAACGATACAGAGAAGATTAGC \\
\hline
\end{tabular}

B, mRNA

\begin{tabular}{lll}
\hline Target & \multicolumn{1}{c}{ Forward sequence (5'-3') } & \multicolumn{1}{c}{ Reverse sequence (5'-3') } \\
\hline Elk4 & CAGCCAGACTGCAAGGTGCTAA & ATCCAGGCCAGACAGAGTGAATG \\
Zeb2 & CCGATCAACCCGTACAAGGA & CTCTCCAGTGATGGTGACCTG \\
Selenop & AGCCATCAAGATCGCTTACTGTG & TGCCCATGTTTGTCATGGTG \\
Tpd52 & GGTGGCAAGATGTGACAGCAA & GATGACTGAGCCAACCGATGAA \\
Xkr8 & TGCAGAGTGGAAATGCCGAATA & TAGTCCAGCAATGCCCACGA \\
Pfkfb3 & GTCGATCACCGACCCTCGTT & CAGTTGAGGTAGCGAGTCAGCTTCT \\
GAPDH & GGCACAGTCAAGGCTGAGAATG & ATGGTGGTGAAGACGCCAGTA
\end{tabular}

miR/miRNA, microRNA; Pfkfb3, 6-phosphofructo-2-kinase/fructose-2,6-biphosphatase 3; Tpd52, tumor protein D52; Xkr8, XK related 8; Elk4, ETS transcription factor ELK4; Selenop, selenoprotein P; Zeb2, zinc finger E-box binding homeobox 2.

miR-223-3p, miR-194-5p, miR-494-3p, miR-429 and miR-29b-3p) and six mRNAs [tumor protein D52, XK related 8 (Xkr8), ETS transcription factor ELK4 (Elk4), 6-phosphofructo-2-kinase/fructose-2,6-biphosphatase 3 (Pfkfb3), selenoprotein P, tumor protein D52 and zinc finger E-box binding homeobox 2].

miRNAs were extracted using the MiPure Cell/Tissue miRNA kit (cat. no. RC201; Vazyme Biotech Co., Ltd.). Purified miRNAs were then reverse transcribed into cDNA using a Mir-X miRNA First-Strand Synthesis kit (cat. no. 638313; Takara Biotechnology Co., Ltd.) according to the manufacturer's protocol and subjected to qPCR using the TB Green Ex Taq II kit (cat. no. RR820A; Takara Biotechnology Co., Ltd.). The thermocycling conditions were as follows: Denaturation at $95^{\circ} \mathrm{C}$ for $10 \mathrm{sec}$; followed by 40 cycles at $95^{\circ} \mathrm{C}$ for $5 \mathrm{sec}$ and $60^{\circ} \mathrm{C}$ for $20 \mathrm{sec}$. The dissociation curve was set at: $95^{\circ} \mathrm{C}$ for $60 \mathrm{sec}, 55^{\circ} \mathrm{C}$ for $30 \mathrm{sec}$ and $95^{\circ} \mathrm{C}$ for $30 \mathrm{sec}$. Reactions were performed on a LightCycler 480 Instrument II (Roche Diagnostics $\mathrm{GmbH}$ ). All reactions were run in triplicate, and data were normalized to the expression levels of U6. Relative quantification of miRNA expression was calculated using the $2^{-\Delta \Delta \mathrm{Cq}}$ method (18).

Total RNA was isolated from cultured cells using RNAiso Plus (cat. no. 9108; Takara Bio, Inc.). A total of $2 \mu \mathrm{g}$ RNA was treated with gDNA Eraser and reverse transcribed into
cDNA using the PrimeScript RT Reagent kit (cat. no. RR047A; Takara Biotechnology Co., Ltd.) according to the manufacturer's protocol. Gene-specific primers were designed and purchased from Sangon Biotech, Co., Ltd. qPCR was performed as described above. Data were analyzed using the $2^{-\triangle \Delta \mathrm{Cq}}$ method with GAPDH as an internal reference gene. All samples were analyzed in triplicate. The primer sequences of the corresponding miRNAs and mRNAs are listed in Table I.

Target gene prediction of DE miRNAs. The miRanda algorithm (19) was used to predict miRNA target genes. The miRanda algorithm comprehensively predicts miRNA target genes based on miRNA-mRNA sequencing matching or energy stability and uses a dynamic programming algorithm to search for regions where miRNAs have complementary sequences with target mRNAs and form stable double-strands.

Construction of a network map of miRNAs and target genes. A global network of DE miRNAs and target genes was constructed using Cytoscape 3.7.2 software (https://cytoscape.org/).

Statistical analysis. Data were analyzed with SPSS 17.0 software (SPSS, Inc.) and presented as the mean \pm SD from at least three separate experiments. Two-tailed independent sample t-test was used to compare the mean values between 
A

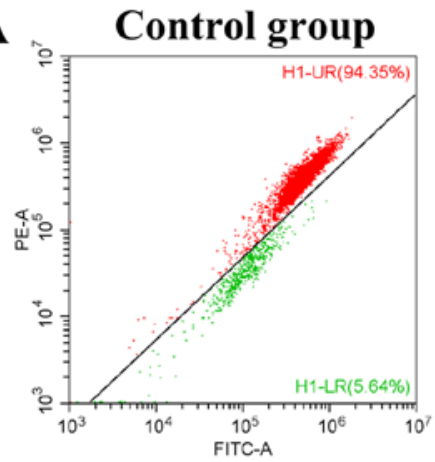

B

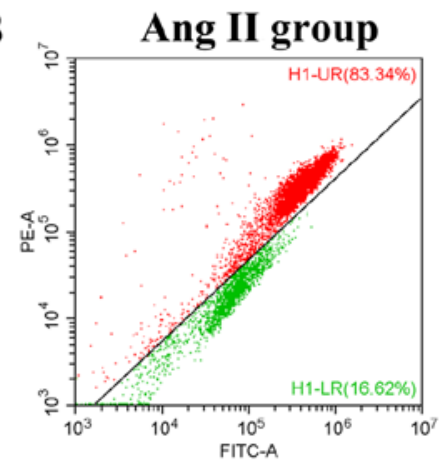

C

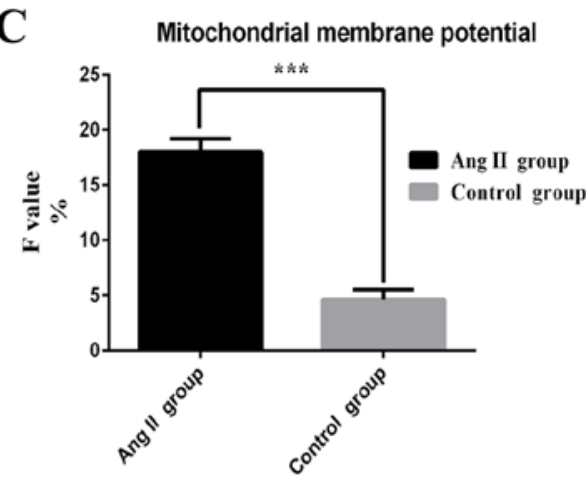

$\mathbf{E}$

D Glycolytic function

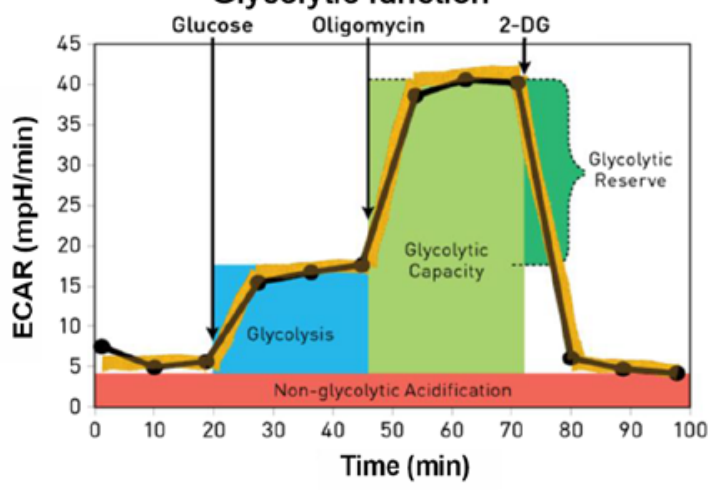

.

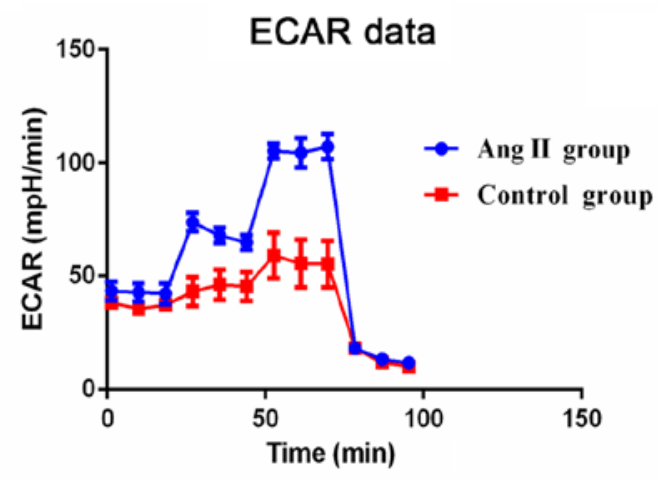

F

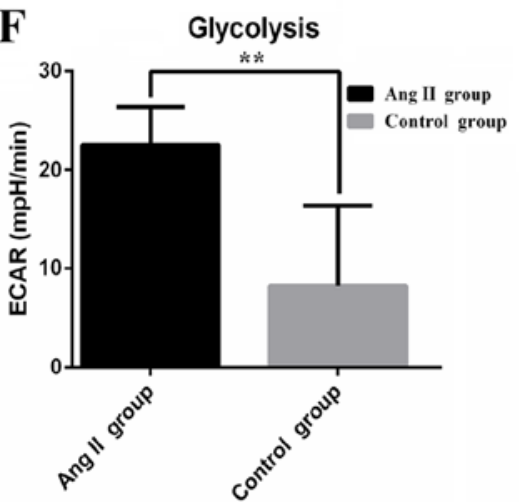

G

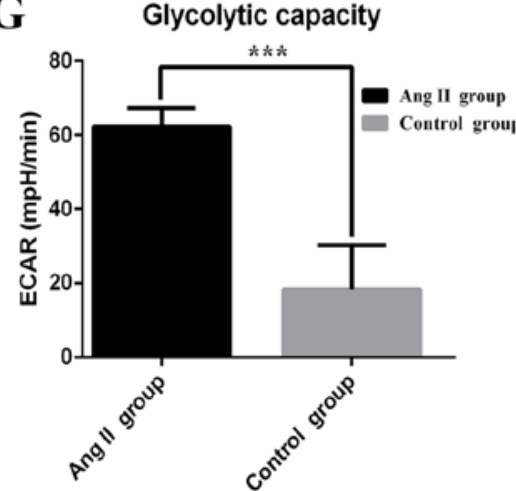

H

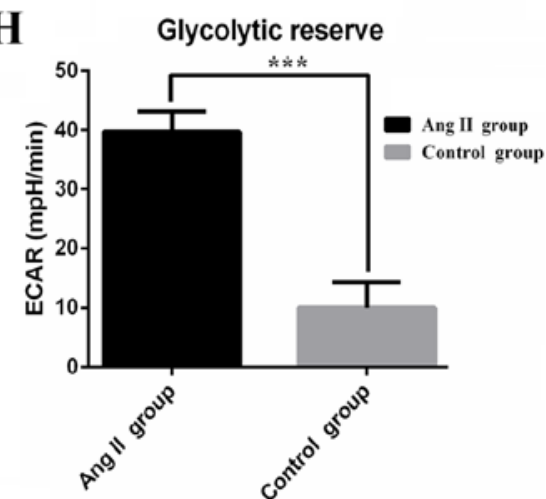

Figure 1. Mitochondrial membrane potentials detected by JC-1 staining. (A) Control group mitochondrial membrane potential results (n=3). (B) Ang II group mitochondrial membrane potential results $(n=3)$. (C) F values showing higher mitochondrial membrane potential in RRAECs treated with Ang II compared with control cells $(n=3)$. ECAR was detected using a Seahorse XF-24 analyzer to assess the glycolytic function of RRAECs. (D) Seahorse XF Glycolysis Stress Test profile of the key parameters of glycolytic activity. The basic value represents the non-glycolytic acid production value of the cells. The injection of glucose represents the rate of glycolysis under basal conditions. After adding oligomycin, mitochondrial ATP production was inhibited and the cells were supplied with oxygen via glycolysis. The subsequent increase in ECAR revealed the cellular maximum glycolytic capacity. The final injection was 2-DG, a glucose analog, that inhibits glycolysis by competitively binding to glucose hexokinase, the first enzyme in the glycolytic pathway. The decreased ECAR represents the glycolytic reserve of the cells. (E) Real-time ECARs obtained from Ang II-induced RRAECs (n=5) and control cells ( $\mathrm{n}=5$ ). (F) ECAR showing higher glycolysis, defined as the maximum ECAR reached by cells after the addition of saturating amounts of glucose, in Ang II-induced RRAECs compared with control cells. (G) ECAR showing higher glycolytic capacity, defined as the maximum ECAR reached by cells following the addition of oligomycin, in Ang II-induced RRAECs compared with control cells. (H) ECAR showing higher glycolytic reserve, defined as the capability of cells to respond to an energetic demand, in Ang II-induced RRAECs compared with control cells. ${ }^{* *} \mathrm{P}<0.01$ and ${ }^{* * *} \mathrm{P}<0.001$. ECAR, extracellular acidification rate; RRAEC, rat renal artery endothelial cell; Ang II, angiotensin II; 2-DG, 2-deoxy-glucose; PE-A, phycoerythrin-area; H1-UR, height1-upper right; H1-LR, height1-lower right.

two groups. $\mathrm{P}<0.05$ was considered to indicate a statistically significant difference.

\section{Results}

Ang II decreases the mitochondrial membrane potential of RRAECs. The F value, defined as the proportion of cells with decreased mitochondrial membrane potential, of cells in the Ang II group was significantly higher compared with the control group $(\mathrm{P}<0.001$; Fig. $1 \mathrm{~A}-\mathrm{C})$. The $\mathrm{F}$ value in the Ang II group was $17.99 \pm 1.23 \%$ while the F value in the control group was $4.64 \pm 0.88 \%$. The results suggested that the normal mitochondrial aerobic respiration of RRAECs was partially inhibited in response to mitochondrial dysfunction.

RRAECs treated with Ang II are highly glycolytic. The Seahorse XF Glycolysis Stress Test provides a standard and comprehensive method to assess the key parameters of glycolytic 
A

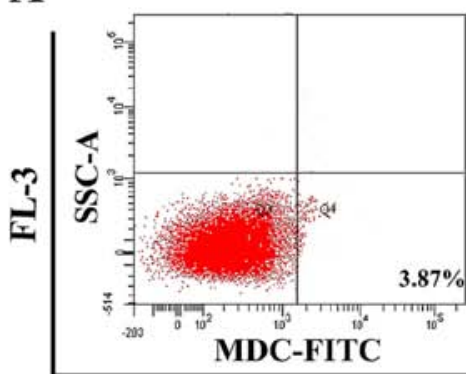

B

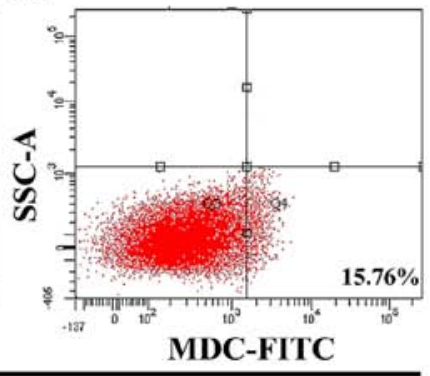

FL-1

D

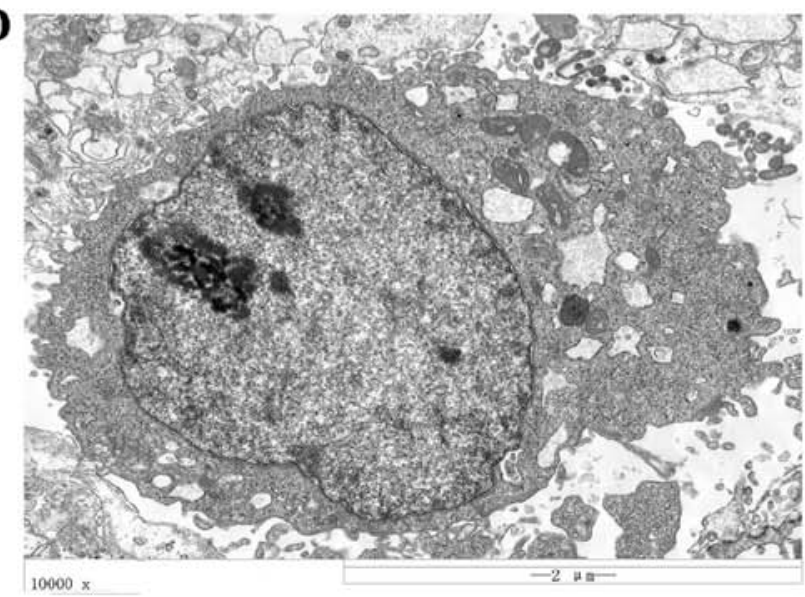

C

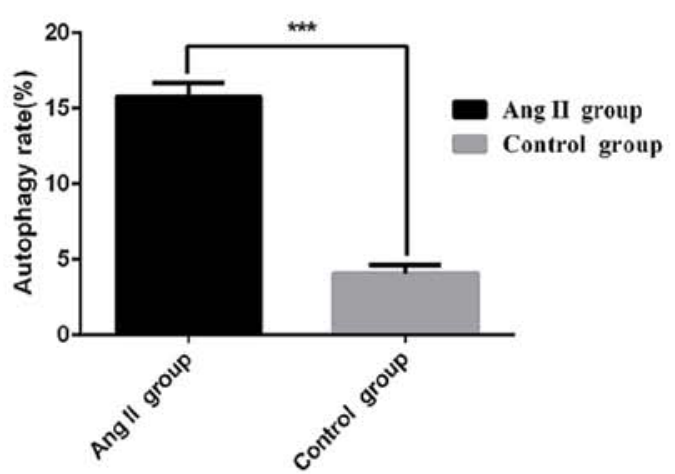

E.

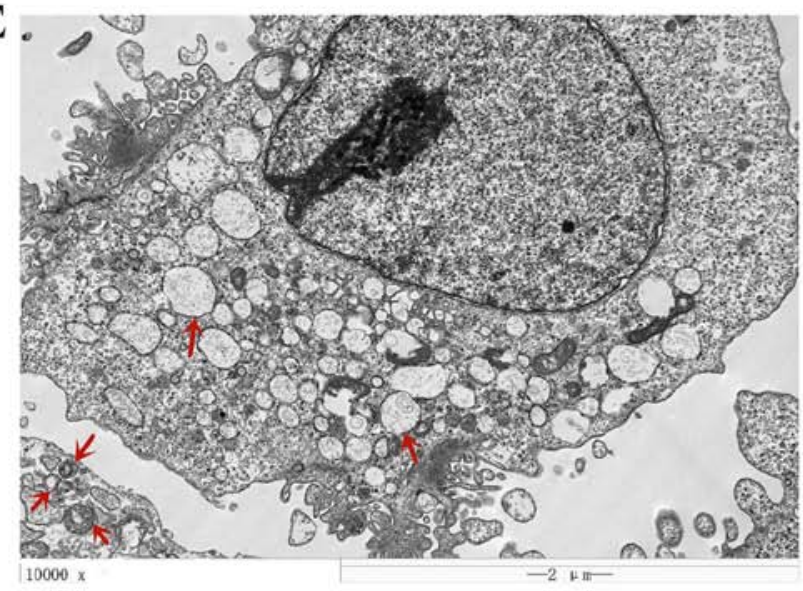

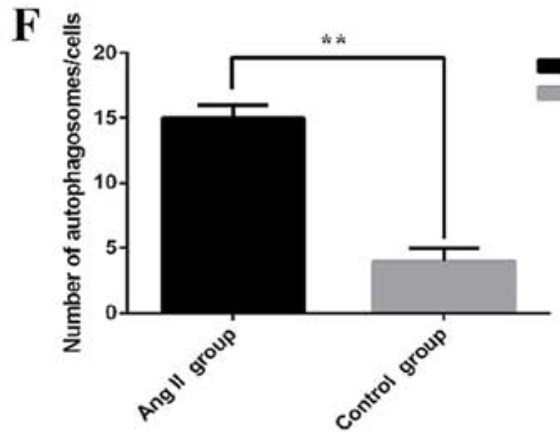

Figure 2. Determination of the autophagy rate and the number of autophagosomes. (A) Autophagy results of the control group (n=3). (B) Autophagy results of the Ang II group ( $n=3)$. (C) Autophagy rate of the control group and the Ang II group. The data from Q4 were analyzed. Autophagosomes were observed by TEM (magnification, $x 10,000)$ in (D) the normal group $(n=3)$ and (E) the Ang II group (n=3). (F) Statistical analysis was performed to quantify the number of autophagosomes in the normal and Ang II groups as shown by TEM. ${ }^{* *} \mathrm{P}<0.01$ and ${ }^{* * *} \mathrm{P}<0.001$. TEM, transmission electron microscopy; Ang II, angiotensin II; MDC, dansylcadaverine; FL; SSC-A.

flux, including glycolysis, glycolytic capacity and glycolytic reserve, by directly measuring the extracellular acidification rate (Fig. 1D and E). The glycolysis value (control group, $8.25 \pm 8.12 \mathrm{mpH} / \mathrm{min}$;AngIIgroup, $22.57 \pm 3.76 \mathrm{mpH} / \mathrm{min} ;$ Fig. $1 \mathrm{~F}$ ), glycolytic capacity value (control group, $18.31 \pm 12.01 \mathrm{mpH} / \mathrm{mi}$; Ang II group, $62.28 \pm 5.03 \mathrm{mpH} / \mathrm{min}$; Fig. 1G) and glycolytic reserve value (control group, $10.06 \pm 4.25 \mathrm{mpH} / \mathrm{min}$; Ang II group, 39.71 $\pm 3.44 \mathrm{mpH} / \mathrm{min}$; Fig. 1H) of RRAECs in the Ang II group were significantly increased compared with the control group. Taken together, these findings suggested that Ang II may cause glycolysis pathway activation.

Ang II promotes autophagy activation in RRAECs. The autophagy rate of RRAECs increased by $\sim 4$-fold $(15.12 \pm 1.45)$ after $24 \mathrm{~h}$ of Ang II treatment compared with the autophagy rate of untreated RRAECs $(3.87 \pm 1.03$; Fig. 2A-C).
Autophagosomes were evaluated in RRAECs cultured with or without Ang II treatment. RRAECs in the control group had a smooth nuclear membrane, a uniform distribution of nuclear chromatin and abundant organelles in the cytoplasm (Fig. 2D). By contrast, TEM showed an increase in the formation of autophagic vesicles in RRAECs treated with Ang II (Fig. 2E). Mitochondria were swollen and vacuolized in the cytoplasm of Ang II-treated cells (Fig. 2E). The number of autophagic corpuscles after $24 \mathrm{~h}$ of Ang II exposure significantly increased compared with the control group (Fig. 2F). Collectively, the data indicated that Ang II might activate the autophagy pathway in RRAECs.

Differential expression profiling of mRNAs and miRNAs. A total of $98,134,568-132,788,030$ raw mRNA reads with clean ratios of $98.315-98.503 \%$ were collected for libraries 
of RRAECs in the Ang II and control groups. After filtering out low-quality raw reads and trimming the $3^{\prime}$ and $5^{\prime}$ adapter sequences, 96,665,811-130,551,102 clean reads were used for genomic alignment (Table II).

A total of 30,446,150-35,655,373 raw miRNA reads with clean ratios of $47-54 \%$ were collected for libraries of RRAECs in the Ang II and control groups. After filtering out low-quality raw reads, $15,852,783-18,500,681$ clean reads were used for genomic alignment (Table III).

DE mRNAs are shown in Fig. 3A and C. After $24 \mathrm{~h}$ of Ang II exposure, a total of 443 DE mRNAs were identified (66 upregulated and 377 downregulated; FC $>1.5$ or $<0.67$; $\mathrm{P}<0.05)$. The mRNA with the most significant difference was 5'-nucleotidase cytosolic IB ( $F C=10.46876 ; \mathrm{P}=0.007145)$. DE miRNAs are shown in Fig. 3B and D. After $24 \mathrm{~h}$ of Ang II treatment, a total of 58 statistically significant DE miRNAs were detected (55 upregulated and 3 downregulated; FC>1.5 or $<0.67 ; \mathrm{P}<0.05)$. The miRNA with the most significant difference was miRNA-7b $\left(\mathrm{FC}=12.44444 ; \mathrm{P}=5.46 \times 10^{-8}\right)$. The 17 upregulated and 3 downregulated miRNAs are presented in Table IV.

$R T-q P C R$ validation of significantly DE miRNAs and DE $m R N A s$. RT-qPCR was performed to validate the RNA-seq data. All nine detected miRNAs were upregulated in the Ang II group compared with the control group (Fig. 4A). The expression results of six mRNAs were consistent with the results of the RNA-seq, with the exception of Elk4 and Xkr8 (Fig. 4B). These data corroborated the results of the miRNA sequencing analysis, indicating that the RNA-seq data was reliable.

Functional analysis of DE mRNAs. GO analysis describes the properties of genes and gene products and provides functional annotation (20). GO covers three aspects of biology: Cellular component (CC), molecular function (MF), and biological process (BP). DE genes were sorted based on the associated P-values, and the top 30 terms are listed in Fig. 5A. After $24 \mathrm{~h}$ of Ang II treatment, the most enriched GO terms associated with endothelial dysfunction in each classification were 'urogenital system development' (GO ID, GO: 0001655; type, BP; $\mathrm{P}=7.49 \times 10^{-8}$ ), 'apical part of cell' (GO ID, GO: 0045177; type, $\mathrm{CC} ; \mathrm{P}=5.66 \times 10^{-14}$ ) and 'oxidoreductase activity, acting on the $\mathrm{CH}-\mathrm{OH}$ group of donors, NAD or NADP as acceptor' (GO ID, GO: 0016616; type, MF; $\mathrm{P}=3.19 \times 10^{-5}$ ). Various enriched biological processes were also observed in the present study, including 'glycolytic process' (Pfkfb3, glycerol-3-phosphate dehydrogenase 1 , fructose-bisphosphatase 1 and peroxisome proliferative activated receptor, gamma, coactivator 1 alpha), 'regulation of nitric oxide biosynthetic process' (aspartate dehydrogenase domain containing, flavin containing dimethylaniline monoxygenase 1 and 3 , and hydroxysteroid 11-b dehydrogenase 1), 'regulation of systemic arterial blood pressure mediated by a chemical' [N-deacetylase and $\mathrm{N}$-sulfotransferase 2, serpin family F member 2, Hyd roxysteroid dehydrogenase11b2 (Hsd11b2) and glutamyl aminopeptidase] (Fig. 5A). The enriched pathways for DE mRNAs were analyzed by KEGG annotation, and the 20 most significantly enriched pathways are listed in Fig. 5B. The main enriched pathways of DE mRNAs after $24 \mathrm{~h}$ of Ang II exposure were 'metabolic pathways' (62 genes), 'glycolysis/gluconeogenesis'
Table II. Raw reads data of pre-processing statistics of mRNAs.

\begin{tabular}{lccc}
\hline Sample & Total reads & Clean reads & Clean ratio (\%) \\
\hline Ang II 1 & $101,301,776$ & $99,721,172$ & 98.43970751 \\
Ang II 2 & $127,551,746$ & $125,429,111$ & 98.33586363 \\
Ang II 3 & $132,788,030$ & $130,551,102$ & 98.31541442 \\
Control 1 & $105,293,284$ & $103,574,371$ & 98.36749987 \\
Control 2 & $104,629,948$ & $102,953,095$ & 98.39734891 \\
Control 3 & $98,134,568$ & $96,665,811$ & 98.50332352
\end{tabular}

Clean reads, number of sequences retained after processing; clean ratio, ratio of clean reads to total reads; Ang II, angiotensin II.

Table III. Raw reads data of preprocessing statistics of microRNAs.

\begin{tabular}{lccc}
\hline Sample & Total reads & Clean reads & Clean ratio (\%) \\
\hline Ang II 1 & $32,376,470$ & $15,852,783$ & 48 \\
Ang II 2 & $35,655,373$ & $18,326,547$ & 51 \\
Ang II 3 & $34,957,905$ & $18,500,681$ & 52 \\
Control 1 & $30,446,150$ & $15,916,405$ & 52 \\
Control 2 & $35,155,302$ & $16,870,836$ & 47 \\
Control 3 & $33,152,924$ & $17,952,659$ & 54 \\
\hline
\end{tabular}

Clean reads, number of sequences retained after processing; clean ratio, ratio of clean reads to total reads; Ang II, angiotensin II.

(8 genes), 'PPAR signaling pathway' (8 genes), 'fatty acid degradation' (5 genes), 'aldosterone-regulated sodium reabsorption' (4 genes), 'pyruvate metabolism' (4 genes), 'AMPK signaling pathway' (7 genes) and 'renin-angiotensin system' (3 genes). The DE mRNAs and DE miRNAs associated with glycolysis are presented in the Tables SI and SII (21-31).

Prediction and functional analysis of DE miRNA targets. miRNA target prediction was performed to explore the potential regulatory roles of miRNAs in RRAECs. Bioinformatics analysis suggested the presence of 4,294 target genes of DE miRNAs after treatment with Ang II for $24 \mathrm{~h}$. As shown in Fig. 5C, the most enriched GO terms of DE miRNA target genes associated with endothelial dysfunction in each class were 'regulation of protein serine/threonine kinase activity' (GO ID, GO: 0071900; type, BP; P=0.000393044), 'vacuolar membrane' (GO ID, GO: 0005774; type, CC; $\mathrm{P}=3.34 \times 10^{-6}$ ) and 'transcriptional activator activity, RNA polymerase II transcription regulatory region sequence-specific binding' (GO ID, GO: 0001228; type, $\mathrm{MF} ; \mathrm{P}=2.40 \times 10^{-6}$ ). KEGG analysis suggested that the pathways of the DE miRNA target genes were mainly enriched in the 'mTOR signaling pathway', 'AMPK signaling pathway', 'autophagy-animal', 'Proximal tube bicarbonate reclamation', 'MAPK signaling pathway', 'insulin resistance' and 'Ras signaling pathway' (Fig. 5D). Fig. 6, which was generated using Pathview package in $\mathrm{R}$, presented DE genes in the mTOR signaling pathway. DE 
A

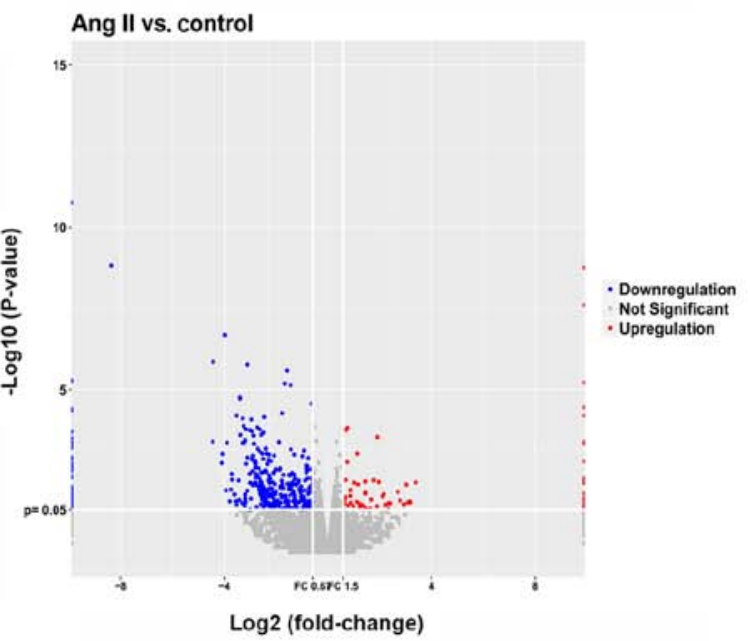

C

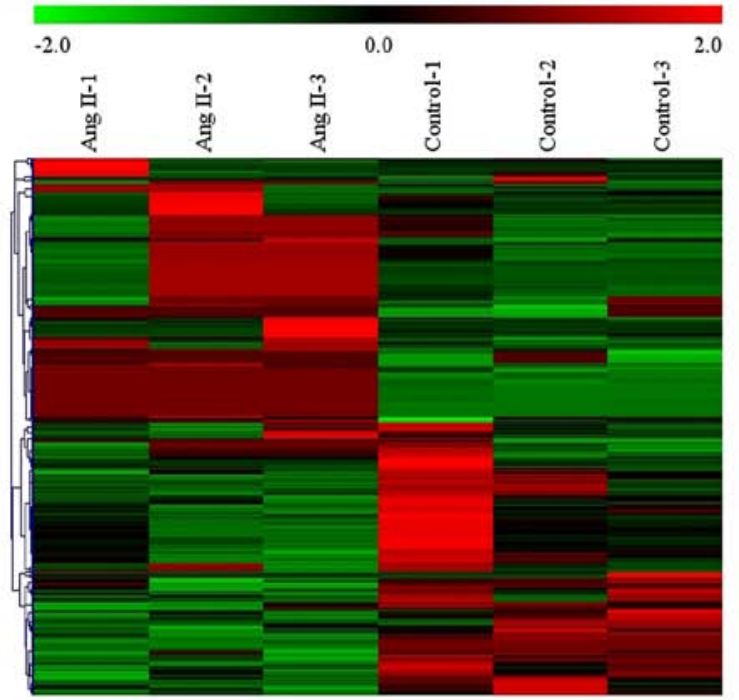

B

Ang II vs. control



D

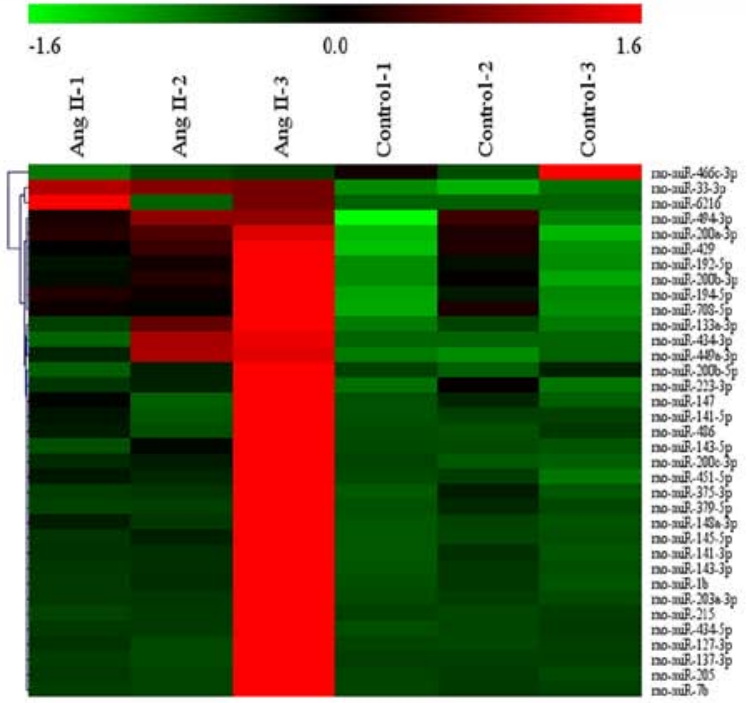

Figure 3. Volcano plots and heatmaps of DE mRNAs and DE miRNAs between the Ang II and control groups. (A) Volcano plot of DE mRNAs between the Ang II groups $(\mathrm{n}=3)$ and control groups $(\mathrm{n}=3)$. (B) Volcano plot of DE miRNAs between the Ang II and control groups. Red represents upregulated DE genes, blue represents downregulated DE genes and grey represents genes that do not differ significantly between the two groups. The abscissa indicates the FC, and an $\mathrm{FC}=1.5$ was used as the screening criterion for differential expression. The ordinate represents the $-\log 10$ (P-value). (C) Heatmap of DE mRNAs between the Ang II and control groups. (D) Heatmap of DE miRNAs between the Ang II and control groups. Black represents 0 and indicates no difference between the groups. Red represents high expression and green represents low expression. DE, differentially expressed; miRNA, microRNA; Ang II, angiotensin II; FC, fold change.

mRNAs and DE miRNAs associated with autophagy are presented in Tables SIII and SIV (32-48).

Network map of DE miRNAs and their target genes. A global network of miRNAs and target genes was constructed to determine the functional interactions between the two types of genes (Fig. 7A). Furthermore, to evaluate the contribution of specific miRNAs, the current study focused on the miRNA-200 family, which was highly and specifically expressed in RRAECs cultured with Ang II and may be a potent regulator of renal artery EC dysfunction. Therefore, a local network map of the miRNA-200 family and their corresponding target genes was constructed (Fig. 7B).

\section{Discussion}

Targeting unique miRNAs which are highly expressed in ECs may be a promising approach in the development of therapeutic tools for diseases associated with endothelial dysfunction. The present study proposed that Ang II activated autophagy pathway in RRAECs, as well as glycolysis pathways to compensate for the lack of the total cellular ATP level caused by mitochondrial respiratory disorder (49). To test this hypothesis, the differential expression of miRNAs and mRNAs in RRAECs after Ang II exposure was profiled in order to extend the list of potential candidate genes involved in hypertensive renal disease. The top 10 DE mRNAs identified in RNA-seq, including matrix metalloproteinase (MMP)-28, anoctamin-1 (ANO1), and myosin light chain kinase 2 (Mylk2), were closely related to EC dysfunction. MMP-28, the newest member of the MMP family, affects the function of hypertensive target organs by impairing the microvascular endothelium (50). MMP-28 overexpression causes EC apoptosis and capillary degeneration, leading to renal arteriosclerosis by directly cleaving the extracellular domain of vascular endothelial growth factor receptor 2 and also increases the expression and activity of MMP-2, causing 
Table IV. Top 10 upregulated and downregulated miRNAs and mRNAs in the Ang II group compared with the control group.

A, miRNA

\begin{tabular}{lccl}
\hline Name & P-value & $\begin{array}{c}\text { Fold } \\
\text { change }\end{array}$ & $\begin{array}{c}\text { Expression } \\
\text { status }\end{array}$ \\
\hline miR-7b & $5.46 \times 10^{-8}$ & 12.44444 & Upregulated \\
miR-127-3p & 0.000344 & 10.4 & Upregulated \\
miR-434-5p & 0.001827 & 9.75 & Upregulated \\
miR-141-5p & 0.032335 & 8 & Upregulated \\
miR-205 & $1.11 \times 10^{-17}$ & 7.074074 & Upregulated \\
miR-215 & $1.82 \times 10^{-14}$ & 6.103448 & Upregulated \\
miR-203a-3p & $3.29 \times 10^{-24}$ & 5.870866 & Upregulated \\
miR-133a-3p & 0.00262 & 5.625 & Upregulated \\
miR-449a-3p & 0.041573 & 5.2 & Upregulated \\
miR-486 & $4.09 \times 10^{-6}$ & 5.172414 & Upregulated \\
miR-143-5p & $9.24 \times 10^{-10}$ & 4.684932 & Upregulated \\
miR-200c-3p & $4.25 \times 10^{-8}$ & 4.228571 & Upregulated \\
miR-1b & $6.25 \times 10^{-14}$ & 4.111801 & Upregulated \\
miR-375-3p & $3.12 \times 10^{-8}$ & 3.721519 & Upregulated \\
miR-148a-3p & $4.84 \times 10^{-10}$ & 3.222222 & Upregulated \\
miR-145-5p & $1.30 \times 10^{-10}$ & 3.207418 & Upregulated \\
miR-451-5p & 0.001682 & 3.151515 & Upregulated \\
5_14943 & 0.029517 & 0.566038 & Downregulated \\
miR-466c-3p & 0.049743 & 0.333333 & Downregulated \\
17_36023_star & 0.020197 & 0.074074 & Downregulated \\
\hline
\end{tabular}

B, mRNA

\begin{tabular}{lccl}
\hline Name & P-value & $\begin{array}{c}\text { Fold } \\
\text { change }\end{array}$ & $\begin{array}{c}\text { Expression } \\
\text { status }\end{array}$ \\
\hline Nt5c1b & 0.007145 & 10.46876 & Upregulated \\
Sectm1b & 0.029084 & 9.008912 & Upregulated \\
Irs3 & 0.008376 & 8.137557 & Upregulated \\
Mmp28 & 0.013961 & 6.498763 & Upregulated \\
Mroh8 & 0.031247 & 5.287269 & Upregulated \\
Ipcef1 & 0.047342 & 5.064618 & Upregulated \\
Ano1 & 0.031278 & 5.028963 & Upregulated \\
Mylk2 & 0.035396 & 4.617663 & Upregulated \\
Olr1387 & 0.016509 & 4.455626 & Upregulated \\
Cldn18 & 0.019303 & 4.307229 & Upregulated \\
Metazoa_SRP & $1.51 \times 10^{-9}$ & 0.003036 & Downregulated \\
Tmprss2 & $1.37 \times 10^{-6}$ & 0.046255 & Downregulated \\
Slc6a19 & 0.001815 & 0.0583 & Downregulated \\
Slc5a8 & 0.000943 & 0.059467 & Downregulated \\
Bhmt2 & $2.04 \times 10^{-7}$ & 0.062865 & Downregulated \\
Anks4b & 0.012484 & 0.065145 & Downregulated \\
Slc26a1 & 0.000423 & 0.0671 & Downregulated \\
Ppargc1a & 0.027021 & 0.072853 & Downregulated \\
Inmt & 0.011903 & 0.075168 & Downregulated \\
Tmem207 & 0.003891 & 0.076696 & Downregulated \\
\hline miRNA_minal & &
\end{tabular}

miRNA, microRNA; Ang II, angiotensin II. damage to renal function (51). MMP-28, which is associated with early hypertensive renal disease, is one of the major risk factors for microalbuminuria in hypertensive patients (52). ANO1 is a newly discovered calcium-activated chloride channel that regulates EC function and participates in vasoconstriction (53). ANO1 overexpression induces an increase in the opening of voltage-dependent calcium channels on the plasma membrane, causing a large amount of $\mathrm{Ca}^{2+}$ influx, whereas overload of intracellular calcium triggers arterial vasoconstriction and participates in the process of elevated blood pressure in vivo (54). A previous study showed that ANO1 was present at high levels in various vascularities of spontaneously hypertensive rats (55). Decreased barrier function caused by EC damage is the starting point of vascular disease, and Mylk2, which encodes myosin light chain kinase (MLCK), increases myosin activity, increases myoglobin-myosin cross-linking, intensifies cell contraction, widens the cell gap through complex signal transduction mechanisms and ultimately increases endothelial permeability (56). Multiple signaling molecules activate MLCK through different pathways to induce endothelial barrier dysfunction (57). The GO terms associated with hypertensive renal disease caused by endothelial dysfunction in the Ang II group included 'glycolytic process' (Pfkfb3, glycerol-3-phosphate dehydrogenase 1, fructose-bisphosphatase 1 and peroxisome proliferative activated receptor, gamma, coactivator 1 alpha), 'regulation of nitric oxide biosynthetic process' (aspartate dehydrogenase domain containing, flavin containing dimethylaniline monoxygenase 1 and 3 , and hydroxysteroid 11-b dehydrogenase 1) and 'regulation of systemic arterial blood pressure mediated by a chemical' [N-deacetylase and $\mathrm{N}$-sulfotransferase 2, serpin family F member 2, Hydroxysteroid dehydrogenase11b2 (Hsd11b2) and glutamyl aminopeptidase]. Numerous reports claimed that reduced bioavailability of NO, as a result of reduced synthesis by ECs, is a key event underlying vascular endothelial damage (58). Hsd11b2, which plays an important regulatory role in the aforementioned biological processes and was significantly downregulated in the Ang II group in the current study, is a newly discovered candidate gene for hypertension that regulates water-salt metabolism and is directly related to blood pressure regulation and the initiation of hypertension $(59,60)$. The KEGG terms enriched by DE mRNAs in the Ang II and control groups were mainly 'glycolysis/gluconeogenesis', 'fatty acid degradation', 'pyruvate metabolism' and the 'renin-angiotensin system'. Experimental studies showed that glutamine and fatty acids were essential substrates, suggesting a critical role for oxidative phosphorylation and mitochondrial respiration in cells (61); however, ECs were reported to rely on glycolysis to a certain degree, especially when intracellular mitochondrial function was abnormal (62). De Bock et al (63) showed that glycolysis levels in ECs were largely comparable to those in tumor cells but higher compared with other healthy cells. In human umbilical vein ECs, the glycolytic flux was $>200$-fold higher $(\sim 1.5 \mu \mathrm{mol}$ glucose $/ \mathrm{h} / \mathrm{mg}$ protein) compared with glucose oxidation, fatty acid oxidation, and glutamine oxidation, partly due to the smaller volume of mitochondria in ECs. The high glycolysis rate maintains lactic acid production and plays a pivotal role in promoting angiogenic signaling. ATP produced by glycolysis is a driver of EC rearrangement in neovascularization (64). The present results demonstrated that Ang II caused mitochondrial dysfunction 

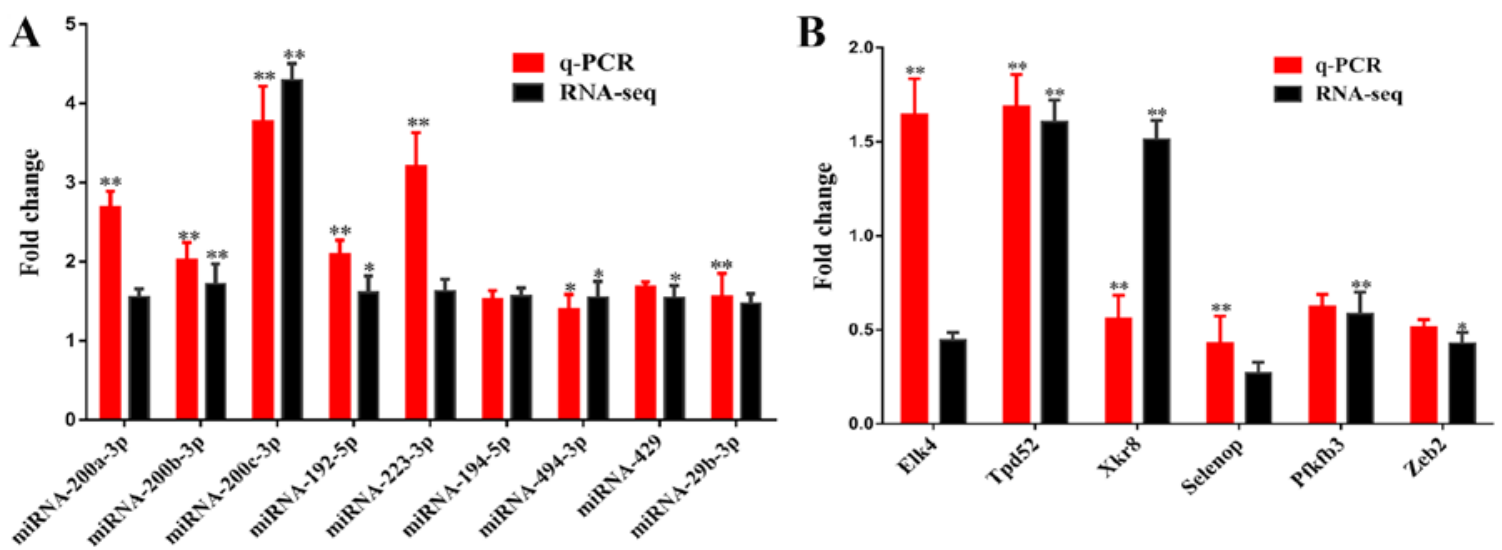

Figure 4. Reverse transcription-quantitative PCR validation ( $n=6)$ and RNA-seq data $(n=3)$ of DE genes. (A) Relative expression of DE miRNAs in the Ang II group compared with the control group in quantitative PCR validation and RNA-seq, respectively. (B) Relative expression of DE mRNAs in the Ang II groups compared with the control groups in qPCR validation and RNA-seq, respectively. ${ }^{* *} \mathrm{P}<0.01$ and ${ }^{*} \mathrm{P}<0.001$. The fold change for each gene (red bar) is the mean of 6 determinations originating from the Ang II groups vs. controls. qPCR, quantitative PCR; miRNA/miR, microRNA; RNA-seq, RNA sequencing; DE, differentially expressed; Pfkfb3, 6-phosphofructo-2-kinase/fructose-2,6-biphosphatase 3; Tpd52, tumor protein D52; Xkr8, XK related 8; Elk4, ETS transcription factor ELK4; Selenop, selenoprotein P; Zeb2, zinc finger E-box binding homeobox 2.

A

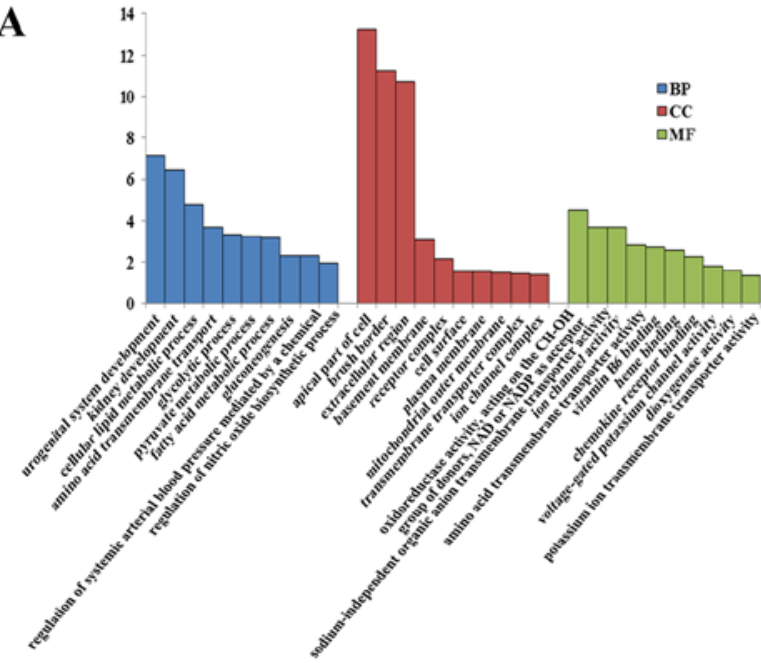

C

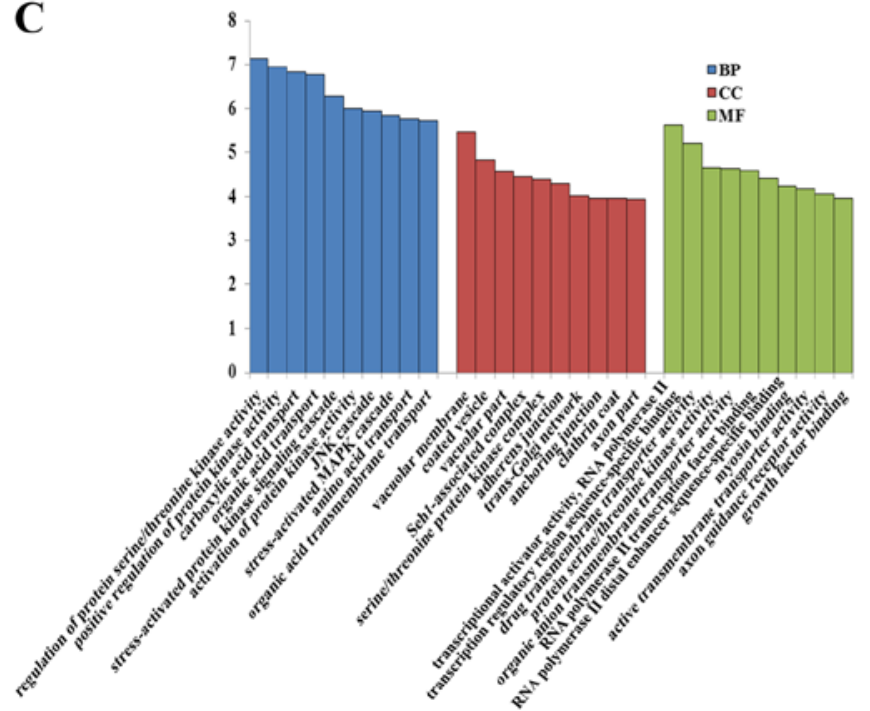

B

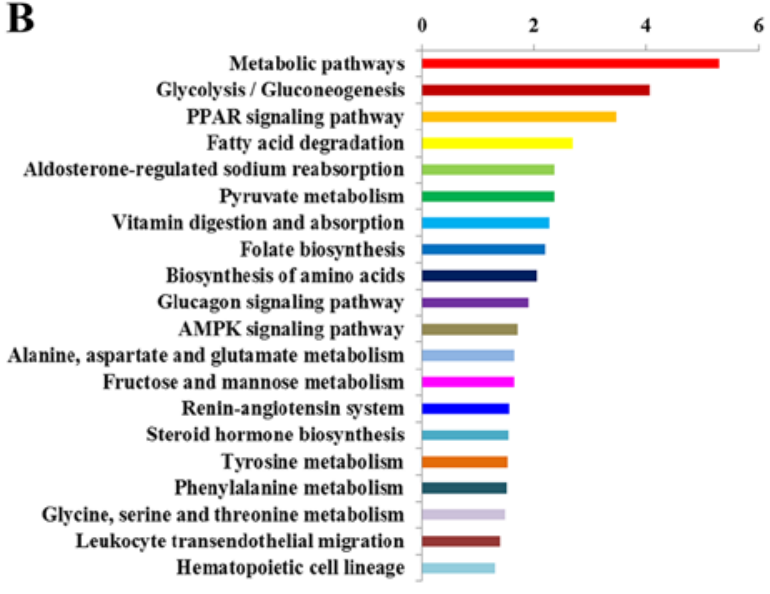

D

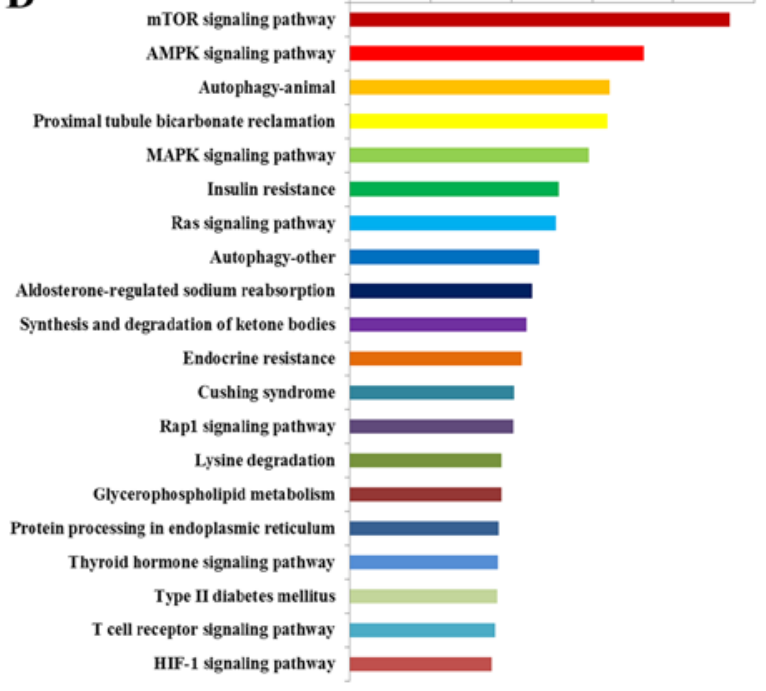

Figure 5. GO and KEGG enrichment analyses of DE mRNAs and miRNAs. (A) GO enrichment of DE mRNAs in the Ang II group compared with the control group. The top $30 \mathrm{GO}$ terms in three categories are summarized. (B) KEGG enrichment of DE mRNAs in the Ang II group compared with the control group. The top 20 pathways of DE mRNAs are summarized. (C) GO enrichment of DE miRNAs in the Ang II group compared with the control group. (D) KEGG enrichment DE miRNAs in the Ang II group compared with the control group. The top 20 pathways of DE miRNA target genes are summarized. GO, Gene Ontology; KEGG, Kyoto Encylopedia of Genes and Genomes; DE, differentially expressed; miRNA, microRNA; Ang II, angiotensin II; BP, biological process; CC, cellular component; MF, molecular function. 


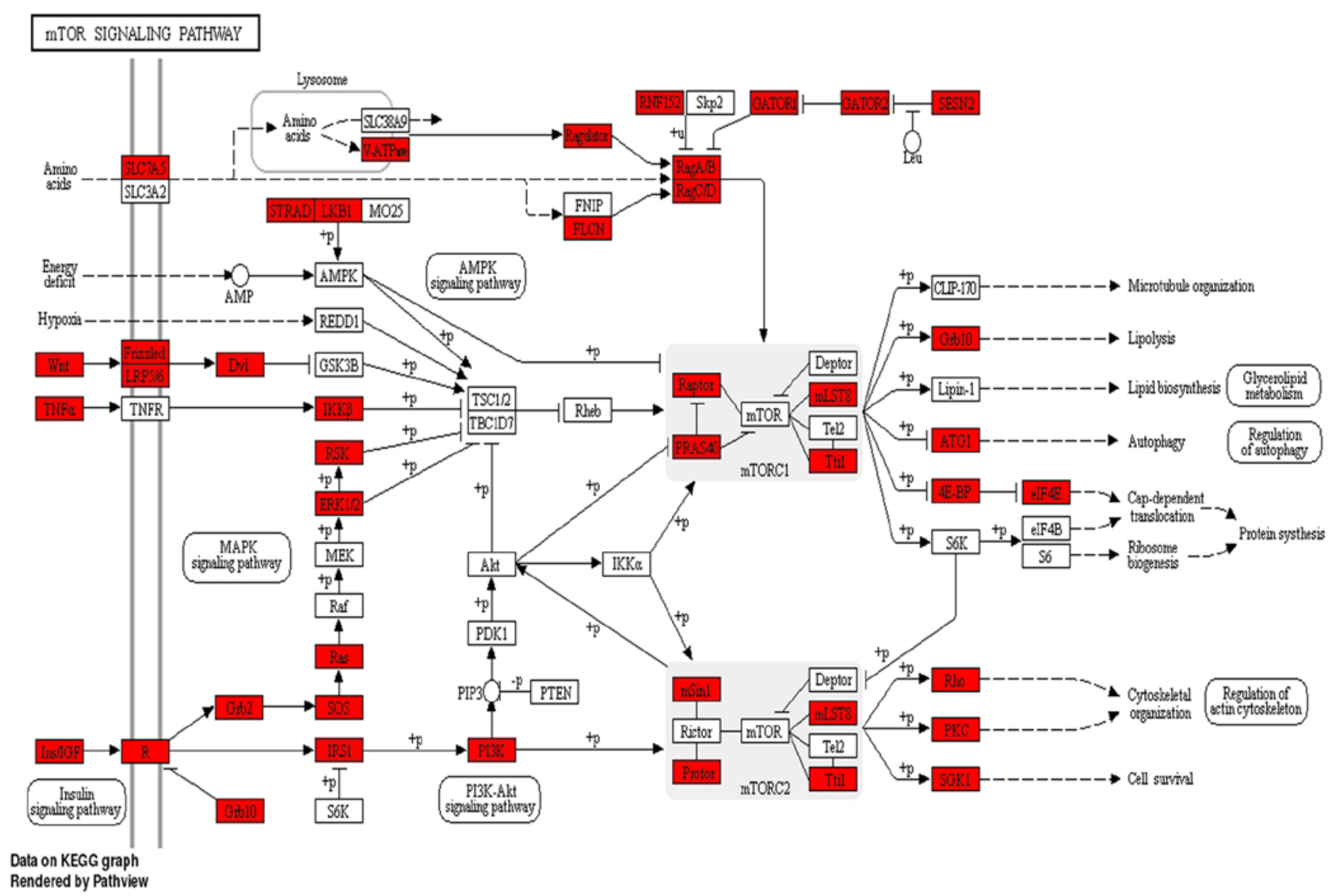

Figure 6. Schematic diagram of the 'mTOR signaling pathway'. Red represents upregulated differentially expressed genes.

and significantly reduced the mitochondrial membrane potential of RRAECs. Therefore, it may be concluded that ECs tend to rely on glycolysis metabolism to compensate for mitochondrial dysfunction. In addition, fatty acid degradation in ECs is directly related to angiogenesis, which regulates biomass synthesis to a certain extent, especially the production of deoxyribonucleotides required for DNA synthesis during EC proliferation (65). Ang II has direct effects on renal vascular ECs, causing vasoconstriction of both afferent and efferent arterioles, which results in the development of both glomerular capillary hypertension and reduced glomerular filtration rates. Ang II has been shown to stimulate the adrenal cortex to secrete aldosterone, leading to increased reabsorption of sodium and water into the distal renal tubules. In addition, Ang II induces vascular NADPH oxidase and ET-1 expression in the kidneys $(66,67)$. Therefore, the relevance of RAS components in the determination of hypertensive nephropathy demonstrated therapeutic implications.

miRNAs are known to play a role in the occurrence and development of hypertensive renal damage. RNA-seq analysis and RT-qPCR results showed that miR-200a-3p, miR-200b-3p, miR-200c-3p and miR-429 were highly expressed in the Ang II group compared with the normal group. These miRNAs are members of the miRNA-200 family, which regulates the expression of transcriptional factors through temporal and spatial patterns during the development of the anterior kidney (68). Therefore, an in-depth analysis of miRNA-200 family members can be performed in a subsequent study based on the constructed network map of the above four miRNAs and their respective target mRNAs. Recent reports demonstrated that miRNA-200c not only promotes epithelial mesenchymal transdifferentiation by inhibiting zinc finger E-box binding to homologous cassette (ZEB) 2 (69), but also upregulates cyclooxygenase 2 expression via ZEB1 to mediate human artery vascular dysfunction (70). The present findings demonstrated that the expression of miRNA-200c was significantly upregulated in the Ang II group compared with the normal group $\left(\mathrm{FC}=4.228571 ; \mathrm{P}=4.25 \times 10^{-8}\right)$. Further insights into the role of miRNA-200 in ECs will improve the understanding of the molecular mechanisms of Ang II-induced renal artery endothelial dysfunction. A previous study has indicated that exogenous platelet miRNA-223 decreases the expression of insulin-like growth factor 1 receptor in HUVEC and thus promotes advanced glycation end product-induced vascular endothelial cell apoptosis (71). Upregulated expression of miRNA-223 in the Ang II group may provide a promising and novel approach to the treatment of hypertension (72). miRNA-494, which increases lipopolysaccharide-induced apoptosis of human proximal tubular epithelial cells by negatively regulating the cyclic AMP-dependent transcription factor ATF-3 gene, was found to mediate the apoptosis of various cells (73). Whether overexpressed miRNA-494 in the Ang II group $(\mathrm{FC}=1.550713$; $\mathrm{P}=0.002353$ ) exerts a similar effect requires further investigation.

As small noncoding RNAs, miRNAs exert their regulatory functions by mRNA degradation or translational inhibition (74). 


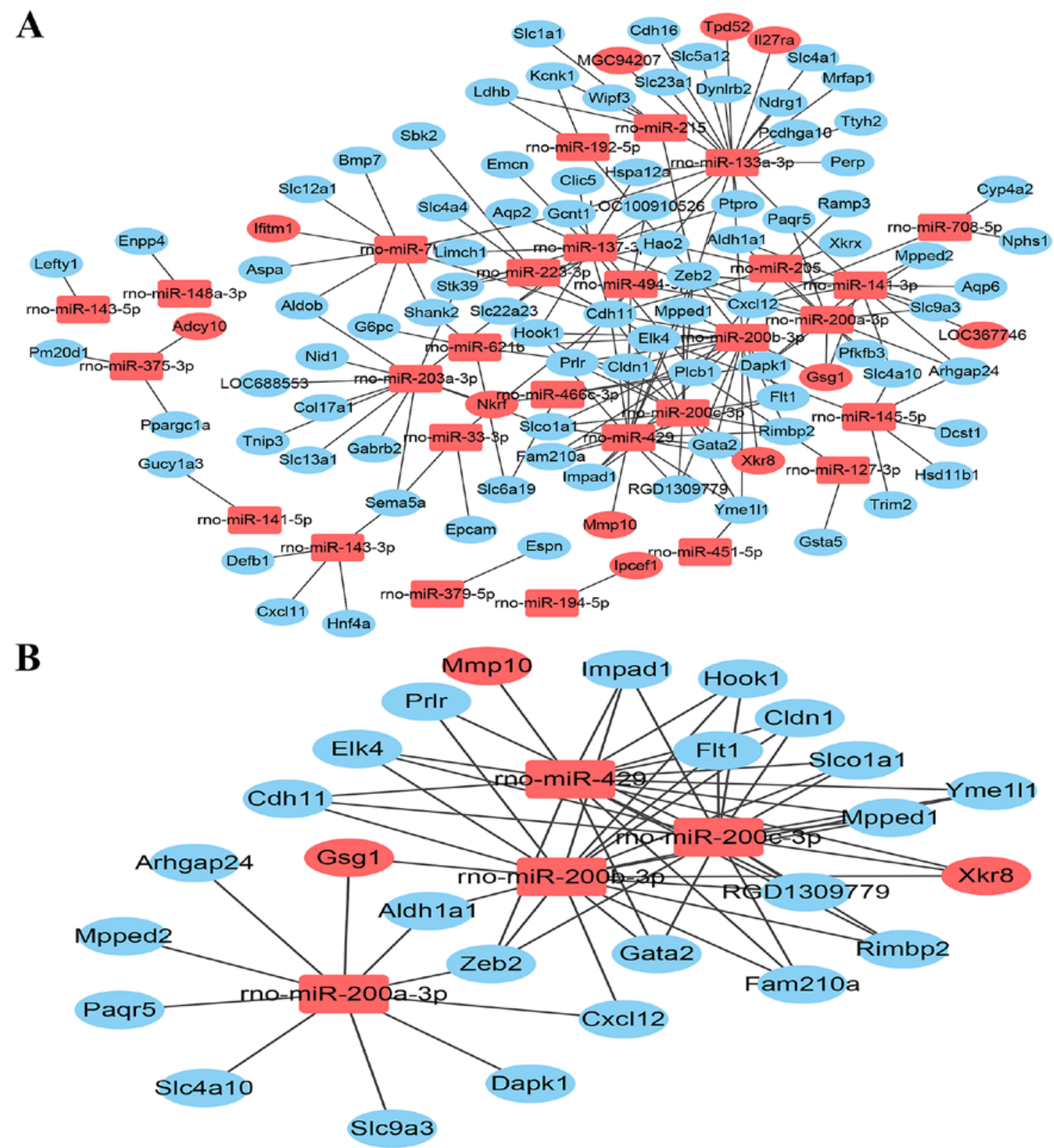

Figure 7. Network maps of DE miRNAs and target genes. (A) Global network of DE miRNAs and their target genes in the Ang II group compared with the control group. (B) Local network map of the miRNA-200 family and their corresponding target genes in the Ang II group compared with the control group. Red represents upregulated DE genes while blue represents downregulated DE genes. DE, differentially expressed; miRNA, microRNA; Ang II, angiotensin II.

In the current study, the target genes of DE miRNAs were mainly enriched in the 'mTOR signaling pathway', 'AMPK signaling pathway' and 'autophagy-animal'. Among these, the mTOR signaling pathway, which regulates the growth and differentiation of cells by sensing the stimulation of growth factors and nutrition, is the main nutrient sensor in the cells and serves a critical role in coordinating intracellular energy metabolism and overall energy levels in vivo $(75,76)$. In addition, mTOR is also a key regulator of autophagy initiation, and mTOR activation inhibits the formation of autophagosomes and negatively regulates autophagy $(77,78)$. Autophagy, which can be regulated by the mTOR pathway, is a biological process in which parts of proteins or organelles encapsulated in a cell membrane are transferred to lysosomes for digestion and degradation to maintain cell homeostasis (79). Furthermore, glycolysis flux, apart from mitochondrial respiratory function, has been shown to be a potential treatment for ischemic kidney damage (80). In ECs, glucose is shuttled through hexokinase into the glycolytic pathway. The product of glycolysis is used not only for the synthesis of glycogen, but also for energy production. A key enzyme for glycolysis, 6-phosphate fructose-2-kinase/fructose-2,6-bisphosphatase 3 is regulated by cellular energy status (the AMPK (81) or growth factor AKT (82) signaling pathways) and participates in protein synthesis regulated by mTOR. The present sequencing results also demonstrated that glycolysis was one of the most important energy metabolism pathways of Ang II-induced renal artery EC dysfunction. Ang II may activate the glycolysis and autophagy pathway in RRAECs through several DE mRNAs or the binding of DE miRNA to its target mRNA. Furthermore, several studies revealed that autophagy may regulate cell glycolysis by selectively degrading hexokinase 2 (83); 
glycolysis in turn plays an essential role in autophagy by limiting superoxide levels and maintaining expression of autophagy genes required for autophagic vesicle maturation (84). Therefore, the effect of the miRNA-mediated mTOR signaling pathway on glycolytic metabolism and autophagy is indicative of the pathological mechanism of Ang II-induced renal artery EC dysfunction.

In conclusion, miRNAs that regulate multiple target genes provide an effective link between regulator target genes and complex biological processes that can be identified through miRNA-mRNA interactions. Further studies investigating the miRNAs and their associated pathway will likely result in the development of novel approaches for the treatment of hypertensive kidney damage.

\section{Acknowledgements}

The authors would like to thank Mr. Yixin Yin of the Shanghai Biotechnology Corporation for technical assistance and constructive suggestions.

\section{Funding}

This study was supported by the National Natural Science Foundation of China (grant no. 81673812).

\section{Availability of data and materials}

The datasets used and/or analyzed during the present study are available from the corresponding author on reasonable request.

\section{Authors' contributions}

YL performed the experiments and drafted the manuscript. YJ designed the study and performed the experiments. WL designed the study, contributed to the discussion and performed important revisions of the article. $\mathrm{CH}$ and $\mathrm{ZQ}$ contributed to data acquisition and software applications. All authors read and approved the final manuscript.

\section{Ethics approval and consent to participate}

Not applicable.

\section{Patient consent for publication}

Not applicable.

\section{Competing interests}

The authors declare that they have no competing interests.

\section{References}

1. Kashiwagi M, Shinozaki M, Hirakata H, Tamaki K, Hirano T, Tokumoto M, Goto H, Okuda S and Fujishima M: Locally activated renin-angiotensin system associated with TGF-beta1 as a major factor for renal injury induced by chronic inhibition of nitric oxide synthase in rats. J Am Soc Nephrol 11: 616-624, 2000
2. Kobori H, Nangaku M, Navar LG and Nishiyama A: The intrarenal renin-angiotensin system: From physiology to the pathobiology of hypertension and kidney disease. Pharmacol Rev 59: 251-287, 2007

3. Klingbeil AU, John S, Schneider MP, Jacobi J, Handrock R and Schmieder RE: Effect of AT1 receptor blockade on endothelial function in essential hypertension. Am J Hypertens 16: 123-128, 2003.

4. Mennuni S, Rubattu S, Pierelli G, Tocci G, Fofi C and Volpe M: Hypertension and kidneys: Unraveling complex molecular mechanisms underlying hypertensive renal damage. J Hum Hypertens 28: 74-79, 2014.

5. Navar LG and Harrison-Bernard LM: Intrarenal angiotensin II augmentation in angiotensin II dependent hypertension. Hypertens Res 23: 291-301, 2000.

6. Geraci G, Mulè G, Mogavero M, Geraci C, D'Ignoti D, Guglielmo C and Cottone S: Renal haemodynamics and severity of carotid atherosclerosis in hypertensive patients with and without impaired renal function. Nutr Metab Cardiovasc Dis 25: 160-166, 2015.

7. Ferraro E, Pulicati A, Cencioni MT, Cozzolino M, Navoni F, di Martino S, Nardacci R, Carrì MT and Cecconi F: Apoptosome-deficient cells lose cytochrome c through proteasomal degradation but survive by autophagy-dependent glycolysis. Mol Biol Cell 19: 3576-3588, 2008.

8. Bartel DP: MicroRNAs: Target recognition and regulatory functions. Cell 136: 215-233, 2009.

9. Zhang R, Su H, Ma X, Xu X, Liang L, Ma G and Shi L: MiRNA let-7b promotes the development of hypoxic pulmonary hypertension by targeting ACE2. Am J Physiol Lung Cell Mol Physiol 316: L547-L557, 2019.

10. Huo KG, Richer C, Berillo O, Mahjoub N, Fraulob-Aquino JC, Barhoumi T, Ouerd S, Coelho SC, Sinnett D, Paradis P and Schiffrin EL: MiR-431-5p knockdown protects against angiotensin II-induced hypertension and vascular injury. Hypertension 73: 1007-1017, 2019.

11. Huang Y, Tang S, Huang C, Chen J, Li J, Cai A and Feng Y: Circulating miRNA-29 family expression levels in patients with essential hypertension as potential markers for left ventricular hypertrophy. Clin Exp Hypertens 39: 119-125, 2017.

12. Xu Z, Zou C, Yu W, Xu S, Huang L, Khan Z, Wang J, Liang G and Wang Y: Inhibition of STAT3 activation mediated by toll-like receptor 4 attenuates angiotensin II-induced renal fibrosis and dysfunction. Br J Pharmacol 176: 2627-2641, 2019.

13. Reers M, Smiley ST, Mottola-Hartshorn C, Chen A, Lin M and Chen LB: Mitochondrial membrane potential monitored by JC-1 dye. Methods Enzymol 260: 406-417, 1995.

14. Biederbick A, Kern HF and Elsässer HP: Monodansylcadaverine (MDC) is a specific in vivo marker for autophagic vacuoles. Eur J Cell Biol 66: 3-14, 1995.

15. Kovács AL and Klionsky DJ: A pioneer in the field of autophagy microscopy: Attila L Kovács. Autophagy 9: 1908-1921, 2013.

16. Liu P, Tseng G, Wang Z, Huang Y and Randhawa P: Diagnosis of T-cell mediated kidney rejection in formalin fixed paraffin embedded tissues using RNA-Seq based machine learning algorithms. Hum Pathol 84: 283-290, 2019.

17. Gu L, Yu J, Wang Q, Xu B, Ji L, Yu L, Zhang X and Cai H: Identification of a 5-lncRNA signature-based risk scoring system for survival prediction in colorectal cancer. Mol Med Rep 18: 279-291, 2018.

18. Livak KJ and Schmittgen TD: Analysis of relative gene expression data using real-time quantitative PCR and the 2(-Delta Delta C(T)) method. Methods 25: 402-408, 2001.

19. Enright AJ, John B, Gaul U, Tuschl T, Sander C and Marks DS: MicroRNA targets in Drosophila. Genome Biol 5: R1, 2003.

20. Popescu M, Keller JM and Mitchell JA: Fuzzy measures on the gene ontology for gene product similarity. IEEE/ACM Trans Comput Biol Bioinform 3: 263-274, 2006.

21. Yu J, Li J, Chen Y, Cao W, Lu Y, Yang J and Xing E: Snail enhances glycolysis in the epithelial-mesenchymal transition process by targeting FBP1 in gastric cancer. Cell Physiol Biochem 43: 31-38, 2017.

22. Cui J, Quan M, Jiang W, Hu H, Jiao F, Li N, Jin Z, Wang L, Wang Y and Wang L: Suppressed expression of LDHB promotes pancreatic cancer progression via inducing glycolytic phenotype. Med Oncol 32: 143, 2015.

23. Tang Y, Zhang Y, Wang C, Sun Z, Li L, Cheng S and Zhou W: Overexpression of PCK1 gene antagonizes hepatocellular carcinoma through the activation of gluconeogenesis and suppression of glycolysis pathways. Cell Physiol Biochem 47: 344-355, 2018 
24. Guo T, Chen T, Gu C, Li B and Xu C: Genetic and molecular analyses reveal G6PC as a key element connecting glucose metabolism and cell cycle control in ovarian cancer. Tumour Biol 36: 7649-7658, 2015

25. Peng SY, Lai PL, Pan HW, Hsiao LP and Hsu HC: Aberrant expression of the glycolytic enzymes aldolase B and type II hexokinase in hepatocellular carcinoma are predictive markers for advanced stage, early recurrence and poor prognosis. Oncol Rep 19: 1045-1053, 2008

26. van Bruggen R, Gualtieri C, Iliescu A, Louicharoen Cheepsunthorn C, Mungkalasut P, Trape JF, Modiano D, Sirima BS, Singhasivanon P, Lathrop M, et al: Modulation of malaria phenotypes by pyruvate kinase (PKLR) variants in a thai population. PLoS One 10: e0144555, 2015.

27. Liu CC, Wang H, Wang WD, Wang L, Liu WJ, Wang JH, Geng QR and Lu Y: ENO2 promotes cell proliferation, glycolysis, and glucocorticoid-resistance in acute lymphoblastic leukemia. Cell Physiol Biochem 46: 1525-1535, 2018.

28. Li X, Jiang Y, Meisenhelder J, Yang W, Hawke DH, Zheng Y, Xia Y, Aldape K, He J,Hunter T, et al: Mitochondria-translocated phosphoglycerate PGK1 functions as a protein kinase to coordinate glycolysis and TCA cycle in tumorigenesis. Mol Cell 61: 705-719, 2016.

29. Kim NH, Cha YH,Lee J,Lee SH, Yang JH, Yun JS, Cho ES,ZZhang X, Nam M, Kim N, et al: Snail reprograms glucose metabolism by repressing phosphofructokinase PFKP allowing cancer cell survival under metabolic stress. Nat Commun 8: 14374, 2017.

30. He J, Jin Y, Chen Y, Yao HB, Xia YJ, Ma YY, Wang W and Shao QS: Downregulation of ALDOB is associated with poor prognosis of patients with gastric cancer. OncoTargets Ther 9 6099-6109, 2016.

31. Liu L, Cai S, Han C, Banerjee A, Wu D, Cui T, Xie G, Zhang J, Zhang X, McLaughlin E, et al: ALDH1A1 contributes to PARP inhibitor resistance via enhancing DNA repair in BRCA2 ovarian cancer cells. Mol Cancer Ther 19: 199-210, 2020.

32. Singh P, Ravanan P and Talwar P: Death associated protein kinase 1 (DAPK1): A regulator of apoptosis and autophagy. Front Mol Neurosci 9: 46, 2016.

33. Riehle C, Wende AR, Sena S, Pires KM, Pereira RO, Zhu Y, Bugger H, Frank D, Bevins J, Chen D, et al: Insulin receptor substrate signaling suppresses neonatal autophagy in the heart. J Clin Invest 123: 5319-5333, 2013.

34. Hou C, Zhu M, Sun M and Lin Y: MicroRNA let-7i induced autophagy to protect $\mathrm{T}$ cell from apoptosis by targeting IGF1R Biochem Biophys Res Commun 453: 728-734, 2014

35. Russell RC, Tian Y, Yuan H, Park HW, Chang YY, Kim J, Kim H, Neufeld TP, Dillin A and Guan KL: ULK1 induces autophagy by phosphorylating Beclin-1 and activating VPS34 lipid kinase. Nat Cell Biol 15: 741-750, 2013

36. Pham HQ, Yoshioka K, Mohri H, Nakata H, Aki S, Ishimaru K, Takuwa N and Takuwa Y: MTMR4, a phosphoinositide-specific 3'-phosphatase, regulates TFEB activity and the endocytic and autophagic pathways. Genes Cells: Jul 2, 2018 (Epub ahead of print).

37. Diao J, Liu R, Rong Y, Zhao M, Zhang J, Lai Y, Zhou Q, Wilz LM, Li J, Vivona S, et al: ATG14 promotes membrane tethering and fusion of autophagosomes to endolysosomes. Nature 520: 563-566, 2015.

38. Zhang D, Xu X and Dong Z: PRKCD/PKC $\delta$ contributes to nephrotoxicity during cisplatin chemotherapy by suppressing autophagy. Autophagy 13: 631-632, 2017.

39. Pascall JC, Rotondo S, Mukadam AS, Oxley D, Webster J Walker SA, Piron J, Carter C, Ktistakis NT and Butcher GW: The immune system GTPase GIMAP6 interacts with the Atg8 homologue GABARAPL2 and is recruited to autophagosomes. PLoS One 8: e77782, 2013

40. Liu H, Mi S, Li Z, Hua F and Hu ZW: Interleukin 17A inhibits autophagy through activation of PIK3CA to interrupt the GSK3B-mediated degradation of BCL2 in lung epithelial cells. Autophagy 9: 730-742, 2013.

41. Jung CH, Jun CB, Ro SH, Kim YM, Otto NM, Cao J, Kundu M and Kim DH: ULK-Atg13-FIP200 complexes mediate mTOR signaling to the autophagy machinery. Mol Biol Cell 20: 1992-2003, 2009.

42. Efeyan A, Zoncu R, Chang S, Gumper I, Snitkin H, Wolfson RL, Kirak O, Sabatini DD and Sabatini DM: Regulation of mTORC1 by the Rag GTPases is necessary for neonatal autophagy and survival. Nature 493: 679-683, 2013.

43. Jung $\mathrm{CH}$, Ro SH, Cao J, Otto NM and Kim DH: mTOR regulation of autophagy. FEBS Lett 584: 1287-1295, 2010.

44. Sakamaki JI and Ryan KM: Autophagy determines the path on the TRAIL to death. Dev Cell 37: 291-293, 2016.
45. Guo Y: Role of HIF-1a in regulating autophagic cell survival during cerebral ischemia reperfusion in rats. Oncotarget 8 : 98482-98494, 2017.

46. Bin BH, Bhin J, Yang SH, Choi DH, Park K, Shin DW, Lee AY, Hwang D, Cho EG and Lee TR: Hyperosmotic stress reduces melanin production by altering melanosome formation. PLoS One 9: e105965, 2014.

47. He C, Zhu H, Zhang W, Okon I, Wang Q, Li H, Le YZ and Xie Z: 7-Ketocholesterol induces autophagy in vascular smooth muscle cells through Nox4 and Atg4B. Am J Pathol 183: 626-637, 2013.

48. Yuan J, Zhang Y, Sheng Y, Fu X, Cheng H and Zhou R: MYBL2 guides autophagy suppressor VDAC2 in the developing ovary to inhibit autophagy through a complex of VDAC2-BECN1-BCL2L1 in mammals. Autophagy 11: 1081-1098, 2015.

49. Wu M, Neilson A, Swift AL, Moran R, Tamagnine J, Parslow D, Armistead S, Lemire K, Orrell J, Teich J, et al: Multiparameter metabolic analysis reveals a close link between attenuated mitochondrial bioenergetic function and enhanced glycolysis dependency in human tumor cells. Am J Physiol Cell Physiol 292: C125-C136, 2007.

50. Androulakis E, Tousoulis D, Papageorgiou N, Latsios G, Siasos G and Stefanadis C: The role of matrix metalloproteinases in essential hypertension. Curr Top Med Chem 12: 1149-1158, 2012.

51. Tran ED, DeLano AF and Schmid-Schönbein GW: Enhanced matrix metalloproteinase activity in the spontaneously hypertensive rat: VEGFR-2 cleavage endothelial apoptosis, and capillary rarefaction. J Vasc Res 47: 423-431, 2010.

52. Williams JM, Zhang J, North P, Lacy S, Yakes M, Dahly-Vernon A and Roman RJ: Evaluation of metalloprotease inhibitors on hypertension and diabetic nephropathy. Am J Physiol Renal Physiol 300: F983-F998, 2011.

53. Allawzi AM, Vang A, Clements RT, Jhun BS, Kue NR, Mancini TJ, Landi AK, Terentyev D, O-Uchi J, Comhair SA, et al: Activation of anoctamin-1 limits pulmonary endothelial cell proliferation via p38-mitogen-activated protein kinase-dependent apoptosis. Am J Respir Cell Mol Biol 58: 658-667, 2018.

54. Heinze C,Seniuk A,Sokolov MV,Huebner AK, KlementowiczAE, Szijártó IA, Schleifenbaum J, Vitzthum H, Gollasch M, Ehmke $\mathrm{H}$, et al: Disruption of vascular $\mathrm{Ca}^{+}$-activated chloride currents lowers blood pressure. J Clin Invest 124: 675-686, 2014

55. Manoury B, Tamuleviciute $A$ and Tammaro $P$ TMEM16A/anoctamin-1 protein mediates calcium-activated chloride currents in pulmonary arterial smooth muscle cells. J Physiol 588: 2305-2314, 2010.

56. Usatyuk PV, Singleton PA, Pendyala S, Kalari SK, He D, Gorshkova IA, Camp SM, Moitra J, Dudek SM, Garcia JG and Natarajan V: Novel role for non-muscle myosin light chain kinase (MLCK) in hyperoxia-induced recruitment of cytoskeletal proteins, NADPH oxidase activation, and reactive oxygen species generation in lung endothelium. J Biol Chem 287: 9360-9375, 2012.

57. Rigor RR, Shen Q, Pivetti CD, Wu MH and Yuan SY: Myosin light chain kinase signaling in endothelial barrier dysfunction. Med Res Rev 33: 911-933, 2013.

58. Torimoto K, Okada Y and Tanaka Y: Type 2 diabetes and vascular endothelial dysfunction. J UOEH 40: 65-75, 2018 (In Japanese).

59. Rahman TJ, Mayosi BM, Hall D, Avery PJ, Stewart PM, Connell JM, Watkins $\mathrm{H}$ and Keavney B: Common variation at the 11-b hydroxysteroid dehydrogenase type 1 gene is associated with left ventricular mass. Circ Cardiovasc Genet 4: 156-162, 2011.

60. White PC, Rogoff D and McMillan DR: Physiological roles of 11 beta-hydroxysteroid dehydrogenase type 1 and hexose-6-phoshate dehydrogenase. Curr Opin Pediatr 20: 453-457, 2008.

61. Spolarics Z, Lang CH, Bagby GJ and Spitzer JJ: Glutamine and fatty acid oxidation are the main sources of energy for Kupffer and endothelial cells. Am J Physiol 261: G185-G190, 1991.

62. Mertens S, Noll T, Spahr R, Krützfeldt A and Piper HM: Energetic response of coronary endothelial cells to hypoxia. Am J Physiol 258: H689-H694, 1990.

63. De Bock K, Georgiadou M, Schoors S, Kuchnio A, Wong BW, Cantelmo AR, Quaegebeur A, Ghesquière B, Cauwenberghs S, Eelen $\mathrm{G}$, et al: Role of PFKFB3-driven glycolysis in vessel sprouting. Cell 154: 651-663, 2013.

64. Polet F and Feron O: Endothelial cell metabolism and tumour angiogenesis: Glucose and glutamine as essential fuels and lactate as the driving force. J Inter Med 273: 156-165, 2013.

65. Schoors S, Bruning U, Missiaen R, Queiroz KC, Borgers G, Elia I, Zecchin A, Cantelmo AR, Christen S, Goveia J, et al: Fatty acid carbon is essential for dNTP synthesis in endothelial cells: Nature 520: 192-197, 2015. 
66. Rüster C and Wolf G: Renin-angiotensin-aldosterone system and progression of renal disease. J Am Soc Nephrol 17: 2985-2991, 2006.

67. Wolf G, Butzmann U and Wenzel UO: The renin-angiotensin system and progression of renal disease: From hemodynamics to cell biology. Nephron Physiol 93: P3-P13, 2003.

68. Tian Z, Greene AS, Pietrusz JL, Matus IR and Liang M: MicroRNA-target pairs in the rat kidney identified by microRNA microarray, proteomic, and bioinformatic analysis. Genome Res 18: 404-411, 2008.

69. Perdigão-Henriques R, Petrocca F, Altschuler G, Thomas MP, Le MT, Tan SM, Hide W and Lieberman J: MiR-200 promotes the mesenchymal to epithelial transition by suppressing multiple members of the Zeb2 and Snail1 transcriptional repressor complexes. Oncogene 35: 158-172, 2016.

70. Filios SR, Xu G, Chen J, Hong K, Jing G and Shalev A: MicroRNA-200 is induced by thioredoxin-interacting protein and regulates Zeb1 protein signaling and beta cell apoptosis. J Biol Chem 289: 36275-36283, 2014.

71. Pan Y, Liang H, Liu H, Li D, Chen X, Li L, Zhang C and Zen K: Platelet-secreted microRNA-223 promotes endothelial cell apoptosis induced by advanced glycation end products via targeting the insulin-like growth factor 1 receptor. J Immunol 192 437-446, 2014

72. Meloche J, Le Guen M, Potus F, Vinck J, Ranchoux B, Johnson I, Antigny F, Tremblay E, Breuils-Bonnet S, Perros F, et al: MiR-223 reverses experimental pulmonary arterial hypertension. Am J Physiol Cell Physiol 309: C363-C372, 2015.

73. Wang X, Zhang X, Ren XP, Chen J, Liu H, Yang J, Medvedovic M, Hu Z and Fan GC: MicroRNA-494 targeting both proapoptotic and antiapoptotic proteins protects against ischemia/reperfusion-induced cardiac injury. Circulation 122: 1308-1318, 2010.

74. Shukla GC, Singh J and Barik S: MicroRNAs: Processing, maturation, target recognition and regulatory functions. Mol Cell Pharmacol 3: 83-92, 2011.

75. Laplante M and Sabatini DM: MTOR signaling in growth control and disease. Cell 149: 274-293, 2012.

76. Shimobayashi $M$ and Hall MN: Making new contacts: The mTOR network in metabolism and signaling crosstalk. Nat Rev Mol Cell Biol 15: 155-162, 2014.
77. Surviladze Z, Sterk RT, DeHaro SA and Ozbun MA: Cellular entry of human papillomavirus type 16 involves activation of the phosphatidylinositol 3-kinase/Akt/mTOR pathway and inhibition of autophagy. J Virol 87: 2508-2517, 2013.

78. Jin Y, Liu S, Ma Q, Xiao D and Chen L: Berberine enhances the AMPK activation and autophagy and mitigates high glucose-induced apoptosis of mouse podocytes. Eur J Pharmacol 794: 106-114, 2017.

79. Bruckova L, Soukup T, Visek B, Moos J, Moosova M, Pavelkova J, Rezabek K, Kucerova L, Micuda S, Brcakova E and Mokry J: Proliferative potential and phenotypic analysis of long-term cultivated human granulosa cells initiated by addition of follicular fluid. J Assist Reprod Genet 28: 939-950, 2011.

80. Rabelink TJ and Carmeliet P: Renal metabolism in 2017: Glycolytic adaptation and progression of kidney disease. Nat Rev Nephrol 14: 75-76, 2018.

81. Garcia D and Shaw RJ: AMPK: Mechanisms of cellular energy sensing and restoration of metabolic balance. Mol Cell 66: 789-800, 2017.

82. Robey RB and Hay N: Akt, hexokinase, mTOR: Targeting cellular energy metabolism for cancer therapy. Drug Discov Today Dis Mech 2: 239-246, 2005.

83. Jiao L, Zhang HL, Li DD, Yang KL, Tang J, Li X, Ji J, Yu Y, Wu RY, Ravichandran S, et al: Regulation of glycolytic metabolism by autophagy in liver cancer involves selective autophagic degradation of HK2 (hexokinase 2). Autophagy 14: 671-684, 2018.

84. Duan L, Perez RE, Davaadelger B, Dedkova EN, Blatter LA and Maki CG: p53-regulated autophagy is controlled by glycolysis and determines cell fate. Oncotarget 6: 23135-23156, 2015.

This work is licensed under a Creative Commons Attribution-NonCommercial-NoDerivatives 4.0 International (CC BY-NC-ND 4.0) License. 